ARTICLE

\title{
The oncogenic transcription factor c-Jun regulates glutaminase expression and sensitizes cells to glutaminase-targeted therapy
}

Michael J. Lukey ${ }^{1}$, Kai Su Greene ${ }^{1}$, Jon W. Erickson ${ }^{2}$, Kristin F. Wilson ${ }^{1}$ \& Richard A. Cerione ${ }^{1,2}$

\begin{abstract}
Many transformed cells exhibit altered glucose metabolism and increased utilization of glutamine for anabolic and bioenergetic processes. These metabolic adaptations, which accompany tumorigenesis, are driven by oncogenic signals. Here we report that the transcription factor c-Jun, product of the proto-oncogene JUN, is a key regulator of mitochondrial glutaminase (GLS) levels. Activation of c-Jun downstream of oncogenic Rho GTPase signalling leads to elevated GLS gene expression and glutaminase activity. In human breast cancer cells, GLS protein levels and sensitivity to GLS inhibition correlate strongly with c-Jun levels. We show that c-Jun directly binds to the GLS promoter region, and is sufficient to increase gene expression. Furthermore, ectopic overexpression of c-Jun renders breast cancer cells dependent on GLS activity. These findings reveal a role for c-Jun as a driver of cancer cell metabolic reprogramming, and suggest that cancers overexpressing JUN may be especially sensitive to GLS-targeted therapies.
\end{abstract}

\footnotetext{
${ }^{1}$ Department of Molecular Medicine, Cornell University, Ithaca, New York 14853, USA. ${ }^{2}$ Department of Chemistry and Chemical Biology, Cornell University, Ithaca, New York 14853, USA. Correspondence and requests for materials should be addressed to R.A.C. (email: rac1@cornell.edu).
} 
T he onset of proliferation imposes a range of biosynthetic and bioenergetic demands on mammalian cells, which are met by a fundamental reprogramming of cellular metabolism ${ }^{1,2}$. The metabolic phenotype of proliferating cells, including cancer cells, typically includes high rates of glucose uptake and glycolysis coupled to lactate secretion (the Warburg effect $)^{3}$, elevated de novo nucleotide biosynthesis ${ }^{4}$, and a high flux of mitochondrial glutamine oxidation ${ }^{5-7}$. Increased nutrient uptake and re-routing of metabolites into anabolic processes are not passive adaptations to the proliferative state, but instead are tightly regulated by the signal transduction pathways and transcriptional networks that promote cell growth and cell cycle progression. Thus, many of the oncogenic signals that lead to cellular transformation directly impact cancer cell metabolism ${ }^{8}$.

Metabolic reprogramming supports the proliferative state but can render cancer cells 'addicted' to certain nutrients, and therefore provides opportunities for novel therapeutic interventions ${ }^{9}$. Some cancer cells show an absolute requirement for an exogenous supply of glutamine, the most abundant amino acid in plasma. Glutamine has many metabolic fates inside the cell, acting as a carbon and nitrogen source for biosynthetic reactions and also contributing to redox homoeostasis ${ }^{5-7}$. However, it is the role of glutamine as an anaplerotic substrate for the tricarboxylic acid (TCA) cycle that underlies the 'glutamine addiction' of many rapidly proliferating cells ${ }^{10,11}$. The sequential conversion of glutamine to glutamate, and then to the TCA cycle intermediate $\alpha$-ketoglutarate $(\alpha-K G)$, provides a mechanism for replenishing carbon that is lost from the cycle to anabolic pathways. The first reaction is catalysed by the mitochondrial enzyme glutaminase (GLS), and the second reaction by glutamate dehydrogenase or by one of several transaminase enzymes.

Two genes, GLS and GLS2, encode glutaminase enzymes in mammals ${ }^{12}$. The GLS gene encodes two splice variants, referred to as kidney-type glutaminase and glutaminase C (GAC), while the GLS2 gene encodes two proteins through a surrogate promoter mechanism, liver-type glutaminase and $\mathrm{GAB}^{12}$. Whereas the GLS2 isozymes are downregulated in several cancers $^{13}$, the GLS isozymes, in particular the GAC splice variant, are frequently upregulated in cancers of the breast ${ }^{14}$, lung $^{15}$, colon ${ }^{16}$, prostate ${ }^{17}$ and brain ${ }^{18}$. Recently, two classes of small-molecule inhibitors of GLS have been identified, based on the lead compounds bis-2-(5-phenylacetamido-1,2,4-thiadiazol2-yl)ethyl sulfide (BPTES) and 968 (refs 19,20). Inhibition of GLS by these molecules, or siRNA-mediated knockdown of GLS, severely impacts the proliferation and/or survival of several cancer cell lines, but does not appear to have detrimental effects on non-tumorigenic cells ${ }^{20,21}$. Thus, there is considerable interest in targeting GLS as a therapeutic strategy against cancer, and the BPTES derivative CB-839 is currently undergoing clinical trials ${ }^{21}$.

One regulator of GLS expression and glutamine catabolism is the transcription factor c-Myc ${ }^{22,23}$. In P493 Burkitt's lymphoma and PC3 prostate cancer cell lines, c-Myc upregulates GLS through an indirect mechanism involving transcriptional repression of micro-RNAs miR-23a/b, which target the $3^{\prime}$-UTR of the GLS transcript and suppress its translation ${ }^{23}$. However, the relationship between $\mathrm{c}$-Myc and glutamine metabolism is complex and tissue specific ${ }^{24}$, and tumour-specific alternative polyadenylation of the GLS transcript can cause a switch of the $3^{\prime}$-UTR, allowing it to escape c-Myc/miR-23-mediated regulation ${ }^{25}$. An apparent uncoupling of c-Myc and GLS has recently been described in human mammary epithelial cells as well as in certain breast cancer cell lines ${ }^{26,27}$.

We previously reported that mitochondrial glutaminase activity becomes elevated during Rho GTPase-mediated cellular transformation ${ }^{20}$. Here we show that the oncogenic transcription factor c-Jun is essential for this signalling outcome, and also acts as a primary regulator of GLS expression in human breast cancer cells. Moreover, we demonstrate that overexpression of the JUN proto-oncogene is sufficient to sensitize breast cancer cells to glutaminase-targeted therapy.

\section{Results}

Rho GTPases can drive glutamine-dependent transformation. We previously reported that oncogenic-Dbl, a guanine nucleotide exchange factor and potent activator of Rho GTPases, signals to upregulate mitochondrial GLS activity in NIH/3T3 cells ${ }^{20}$. This is an essential event for maintaining Dbl-induced cellular transformation. To explore further the signalling connections that link Rho GTPases with GLS, we utilized an inducible, tetracycline-off, system to control the expression of oncogenic$\mathrm{Dbl}$ in mouse embryonic fibroblasts (MEFs). When doxycycline $\left(0.6 \mu \mathrm{g} \mathrm{ml}^{-1}\right)$ is present in the culture medium, HA-tagged oncogenic-Dbl is undetectable by western blot analysis of wholecell lysates. Removal of doxycycline triggers a robust expression of oncogenic-Dbl within $10 \mathrm{~h}$ that remains elevated through $72 \mathrm{~h}$ (Fig. 1a). This is accompanied by a corresponding increase in GLS protein levels, which peak $24-48 \mathrm{~h}$ following induction (Fig. 1a). We isolated mitochondria from uninduced and induced MEFs and assayed the preparations for glutaminase activity as described previously ${ }^{28}$. This confirmed that induction of oncogenic-Dbl results in elevated glutaminase activity (Fig. 1b). We then tested whether GLS was upregulated at the transcriptional level. Cells that were either uninduced or induced $(24 \mathrm{~h})$ were analysed by real-time PCR (RT-PCR), which revealed that induction of oncogenic-Dbl expression leads to an $\sim 12$-fold increase of the GLS transcript (Fig. 1c).

Induction of oncogenic-Dbl caused MEFs to acquire transformed characteristics, including greatly increased saturation density, and the ability to proliferate in low serum $(0.5 \%$ fetal bovine serum (FBS)) medium and to undergo anchorage-independent growth. Since these changes were accompanied by an increase in GLS levels, we tested whether cellular transformation was dependent on an exogenous supply of glutamine, and/or on GLS enzymatic activity. After 8 days of culture in medium supplemented with $10 \%$ FBS $\pm 4 \mathrm{mM}$ glutamine, saturation density was assessed by fixing and staining cells with crystal violet (Fig. 1d). Prior to fixation, cells were imaged at $\times 100$ magnification (Supplementary Fig. 1a). The increased saturation density of cells expressing oncogenic-Dbl was completely abolished in glutamine-free medium, but could be partially rescued by supplementation with a cell-permeable analogue of $\alpha$-ketoglutarate (dimethyl- $\alpha-\mathrm{KG}$ ), a downstream metabolite of the GLS reaction. We compared cell viability under high-glutamine $(4 \mathrm{mM})$ and low-glutamine $(0.2 \mathrm{mM})$ conditions, and found that uninduced cells remained fully viable following glutamine depletion, whereas induced cells showed an $\sim 5$-fold increase in cell death (Fig. 1e). The proliferation rate of both uninduced and induced cells decreased on glutamine deprivation, although the inhibition was $\sim 2$-fold greater in induced cells (Fig. 1f).

We then used BPTES ${ }^{19}$, a small-molecule inhibitor of GLS, to test whether MEFs transformed by oncogenic-Dbl were dependent on GLS activity. Saturation density of induced cells was decreased by BPTES treatment in a dose-dependent manner, and partially rescued by supplementation with dimethyl- $\alpha-K G$ (Fig. 1g and Supplementary Fig. 1b). Cell proliferation assays illustrated the remarkable difference in BPTES sensitivity between uninduced and induced MEFs (Fig. 1h). Proliferation of induced cells was potently inhibited by BPTES with an $\mathrm{IC}_{50}$ of $8 \mu \mathrm{M}$, whereas uninduced cells were unaffected even by $20 \mu \mathrm{M}$ BPTES. Oncogenic-Dbl-induced cells, but not uninduced cells, were capable of anchorage-independent growth. This too was completely blocked by BPTES treatment (Fig. 1i). 
a

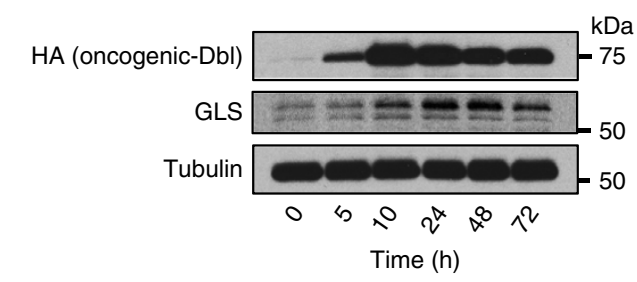

b

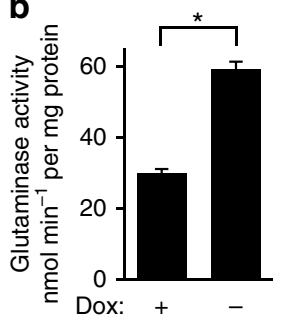

C

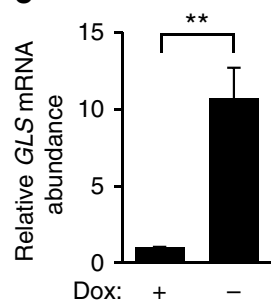

d

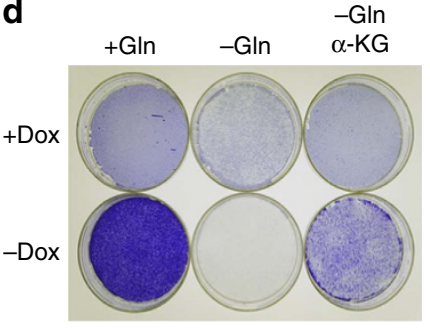

$9 \quad \begin{aligned} & + \text { BPTES } \\ & + \text { BPTES } \\ & +\alpha-K G\end{aligned}$

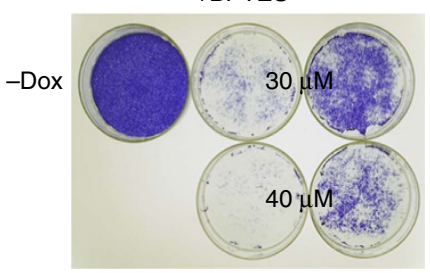

e

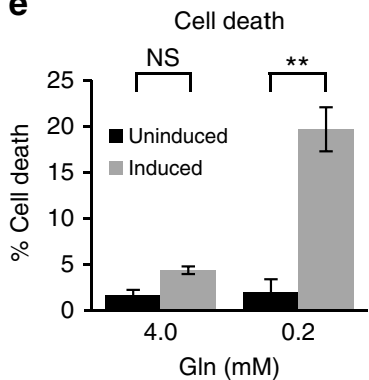

f

Cell proliferation

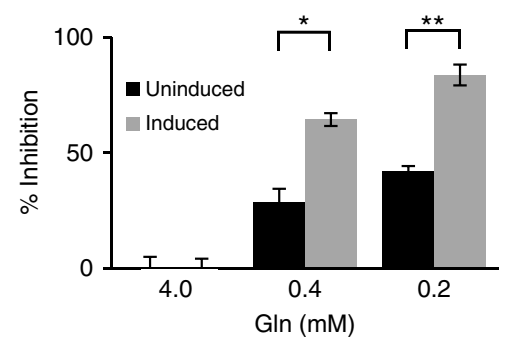

h

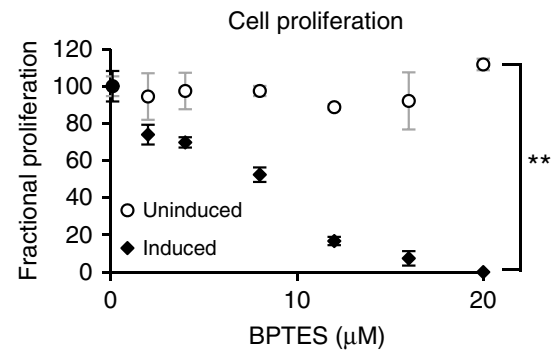

i

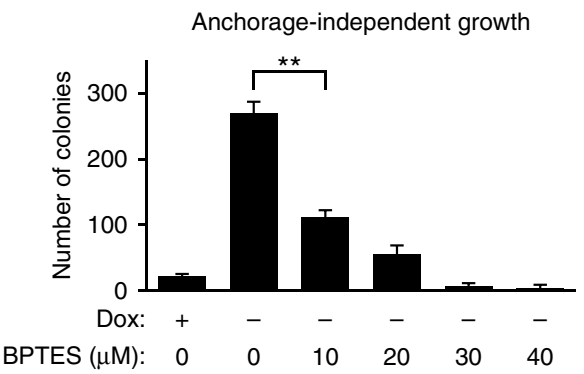

Figure 1 | Glutamine-dependent transformation by oncogenic-Dbl. (a) Western blot analysis showing timecourse of oncogenic-Dbl expression in an inducible MEF system, and downstream elevation of GLS levels. Cells were induced by plating in doxycycline-free growth medium, and samples were collected at time-points up to $72 \mathrm{~h}$. (b) Glutaminase activity assay using mitochondria isolated from MEFs in which oncogenic-Dbl expression was either uninduced ( + Dox) or induced for $24 \mathrm{~h}$ ( - Dox). Activity is expressed per $\mathrm{mg}$ of total cellular protein, and data presented are the mean \pm s.d. of triplicate assays. (c) RT-PCR analysis of uninduced and induced ( $24 \mathrm{~h}$ ) MEFs, showing relative levels of the GLS transcript. The data presented are the RQ values, with error bars marking RQ max and RQ min, from triplicate reactions. (d) Saturation density analysis showing the effect of glutamine withdrawal on oncogenic-Dbl inducible MEFs. Dishes of uninduced ( + Dox) and induced ( - Dox) cells cultured in $4 \mathrm{mM}$ glutamine, or in glutamine-free medium $\pm 2 \mathrm{mM}$ dimethyl $\alpha$-ketoglutarate, were fixed and then stained with crystal violet. (e) Cell death analysis for uninduced or induced cells after 6 days culture in 4.0 or $0.2 \mathrm{mM}$ glutamine. Data presented are the mean \pm s.d. of triplicate assays. (f) Cell proliferation assays showing the effect of glutamine depletion on proliferation of uninduced and induced MEFs over 6 days. Data presented are the mean \pm s.d. of triplicate assays. (g) Saturation density analysis showing the effect of the GLS inhibitor BPTES on oncogenic-Dbl induced MEFs. Induced ( - Dox) cells cultured in the absence or presence of BPTES (30 or $40 \mu \mathrm{M}) \pm 2 \mathrm{mM}$ dimethyl $\alpha$-ketoglutarate were fixed and then stained with crystal violet. (h) BPTES dose curves showing the effect of different BPTES concentrations on proliferation over 6 days of uninduced or induced MEFs. Fractional proliferation relative to untreated cells is shown. Assays were carried out in $10 \%$ FBS medium, and data presented are the mean \pm s.d. of triplicate assays. (i) Anchorage-independent growth assay for uninduced ( + Dox) cells, and for induced ( - Dox) cells cultured under increasing BPTES concentrations. Data presented are the mean \pm s.d. of triplicate assays. Differences were analysed with Student's $t$-test. ${ }^{\star} P<0.05,{ }^{\star \star} P<0.01$.

Rho GTPases signal to c-Jun to upregulate GLS expression. The results above show that oncogenic-Dbl signals to upregulate GLS at the transcript and protein level, and that cellular transformation driven by oncogenic-Dbl is dependent on GLS activity and an exogenous supply of glutamine. We next wanted to identify the signalling pathway downstream of the Rho GTPases that was responsible for regulating GLS expression. We first used phospho-specific antibodies to assess the effects of 

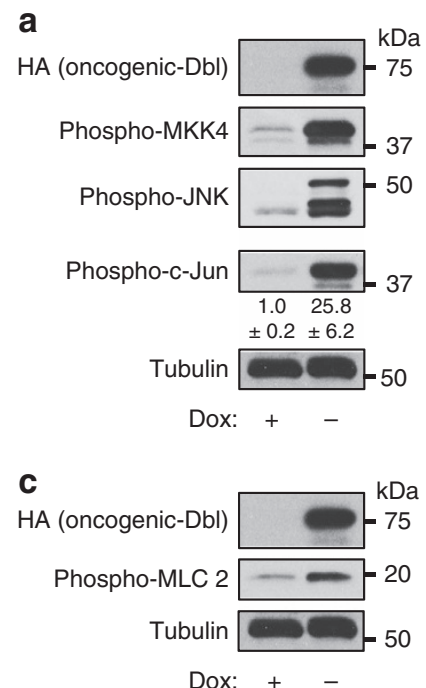
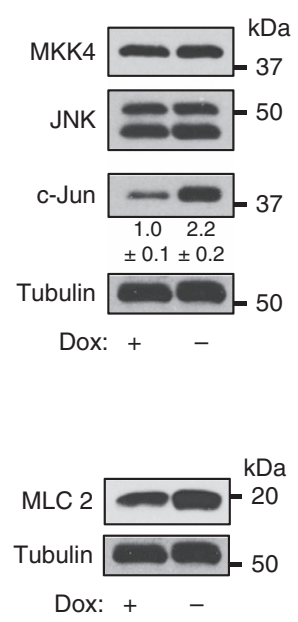

b

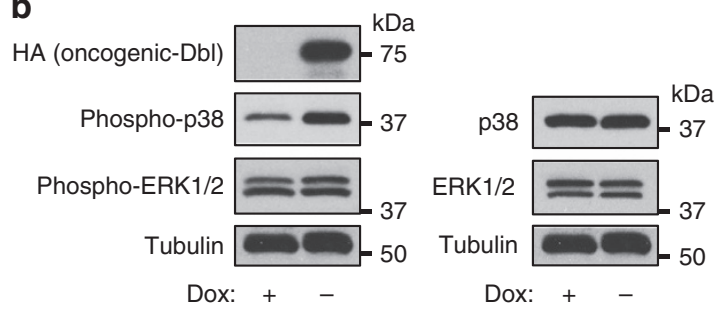

d

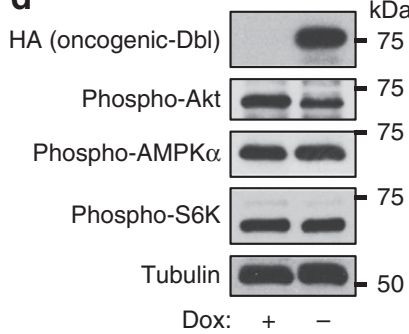

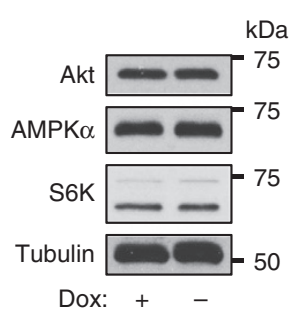

Figure 2 | Oncogenic-Dbl potently activates the MKK4-JNK-c-Jun signalling axis in MEFs. Western blot analysis of whole-cell lysates of MEFs in which oncogenic-Dbl expression was either uninduced (+ Dox) or induced for $24 \mathrm{~h}$ ( - Dox) under low-serum (0.5\% FBS) conditions. The extent of activation of different signalling pathways was assessed using phospho-specific antibodies that recognize activated kinases or their downstream phosphorylation targets (left panels). Any changes in total protein levels were also assessed (right panels). (a) The MAPK signalling axis involving MKK4-JNK-c-Jun is potently activated downstream of oncogenic-Dbl. Total c-Jun levels are also elevated, consistent with previous reports showing auto-regulation of JUN expression. (b) The MAPK p38 is moderately activated, whereas ERK is only slightly activated on oncogenic-Dbl induction. (c) ROCK activity, as read-out by MLC 2 phosphorylation, is activated on induction. (d) Akt is slightly inhibited, and AMPK and mTORC1 activity (the latter read-out by S6K phosphorylation) are largely unaffected by oncogenic-Dbl induction. Relative densitometry data are the mean $\pm \mathrm{s}$.d. of triplicate blots.

oncogenic-Dbl induction on key cellular signalling proteins. Here, and throughout the study, low-serum conditions $(0.5 \%$ FBS $)$ were used for signalling experiments to minimize 'background' cellular signalling activity. Western blot analysis of whole-cell lysates revealed an extremely potent activation of the MKK4/c-Jun $\mathrm{N}$-terminal kinase (JNK) signalling axis on induction $(24 \mathrm{~h})$, and downstream activating phosphorylation of the oncogenic transcription factor c-Jun (Fig. 2a). c-Jun is known to autoregulate its own gene expression in $\mathrm{MEFs}^{29,30}$ and, consistent with this, we found that total c-Jun levels increased as well as phosphorylated c-Jun levels (2-fold and 26-fold, respectively) (Fig. 2a and Supplementary Fig. 2a). The JNK kinase MKK7 and the Jun-family members JunB and JunD were expressed at low levels, and phosphorylated forms could not be detected (Supplementary Fig. 2b,c). Another target of MKK4, p38, was phosphorylated downstream of oncogenic-Dbl, whereas the activation status of ERK showed only a subtle change (Fig. 2b). Rho-associated coiled-coil containing protein kinase (ROCK) was likely activated, as indicated by increased phosphorylation of its substrate myosin light chain 2 (MLC 2) (Fig. 2c). Consistent with earlier reports that ROCK signals to inhibit $\mathrm{Akt}^{31}$, a modest decrease in Akt phosphorylation at residue T308 occurred on induction (Fig. 2d), and this could be rescued by inhibition of ROCK (Supplementary Fig. 2d). The mTORC1-S6K signalling axis showed little change on oncogenic-Dbl induction, and we also detected no change in the phosphorylation level of AMPK $\alpha$ (Fig. 2d).

Since ROCK, p38 and JNK signalling were all activated downstream of oncogenic-Dbl, we tested whether selective inhibition of these kinases (by the small-molecule inhibitors Y-27632, SB203580 and SP600125, respectively) impacted the ability of oncogenic-Dbl to upregulate GLS. Cells were induced for $48 \mathrm{~h}$, either in the absence or presence of $10 \mu \mathrm{M}$ of each inhibitor, and whole-cell lysates were then analysed by western blot. Treatment of induced cells with the ROCK inhibitor Y-27632 or the p38 inhibitor SB203580 did not significantly impact GLS levels, whereas the JNK inhibitor SP600125 largely blocked the upregulation of GLS (Fig. 3a). Although rapamycinsensitive mTORC1 can influence GLS levels by increasing MYC translation $^{32}$, we found that regulation of GLS downstream of the Rho GTPases was independent of mTORC1 activity (Fig. 3b), and changes in c-Myc (and phospho-c-Myc) levels did not fully correspond to changes in GLS (Supplementary Fig. 2e). Treatment of induced cells with the c-Myc inhibitor 10058-F4 severely impacted viability in low-serum (0.5\% FBS) culture medium (no viable cells remained after $24 \mathrm{~h}$ treatment with $10 \mu \mathrm{M} 10058-\mathrm{F} 4)$, and it is possible that c-Myc contributes to the regulation of GLS expression by Rho GTPases under these conditions. In high-serum (10\% FBS) medium, treatment with 10058-F4 at concentrations up to $60 \mu \mathrm{M}$ had little impact on GLS levels in induced cells (Supplementary Fig. 2f), whereas inhibition of JNK by $10 \mu \mathrm{M}$ SP600125 still suppressed GLS expression (Supplementary Fig. 2e).

Consistent with the effects on GLS protein levels, treatment with the JNK inhibitor SP600125 $(10 \mu \mathrm{M})$ completely blocked the increase in mitochondrial glutaminase activity that occurs downstream of oncogenic-Dbl signalling (Fig. 3c). Because JNK directly phosphorylates activator protein 1 (AP-1) family transcription factors including the archetypal substrate c-Jun, leading to increased transcriptional activity, we tested whether blockade of JNK signalling affected GLS transcript levels. The $\sim 12$-fold increase in GLS mRNA that occurs following oncogenic-Dbl induction was completely abolished by $10 \mu \mathrm{M}$ SP600125 (Fig. 3d). To test the hypothesis that the effects of JNK inhibition were mediated by suppression of c-Jun transcriptional activity, we treated induced cells with $10 \mu \mathrm{M}$ SR11302, a selective 
a

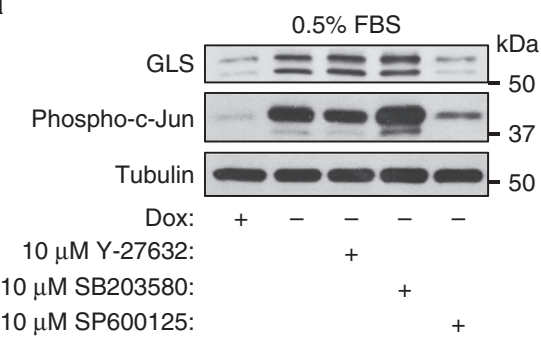

b

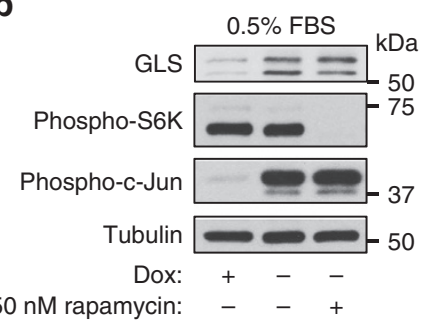

C

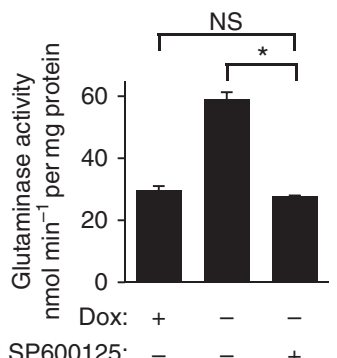

d

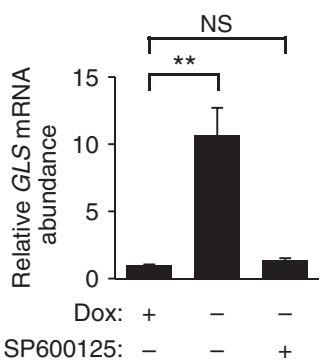

e

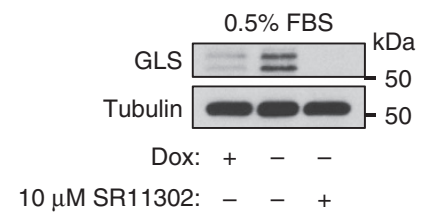

f

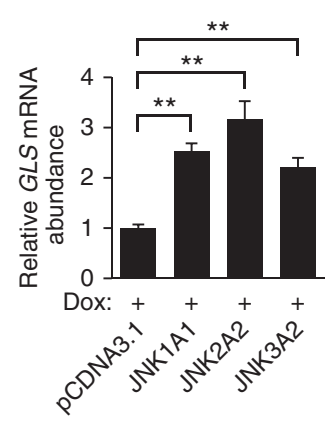

h

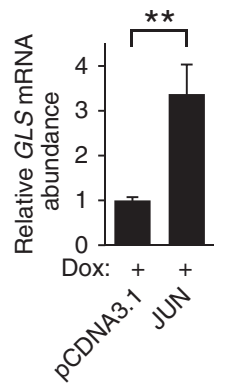

g

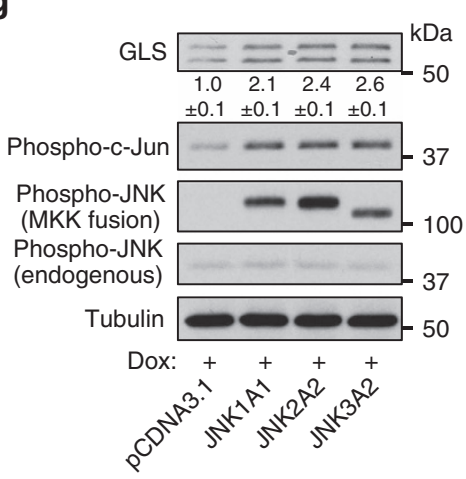

i

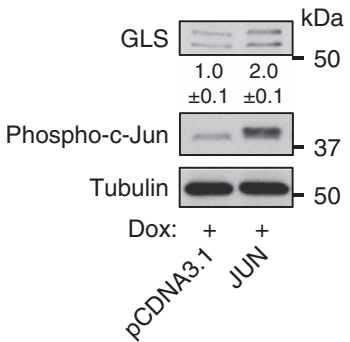

Figure 3 | Oncogenic-Dbl signals to c-Jun to upregulate GLS in MEFs. (a) Western blot analysis of whole-cell lysates of uninduced ( + Dox) or induced for $48 \mathrm{~h}$ ( - Dox) MEFs $\pm 10 \mu \mathrm{M}$ selective inhibitors of ROCK (Y-27632), p38 (SB203580) or JNK (SP600125). Inhibition of JNK largely blocks the upregulation of GLS downstream of oncogenic-Dbl. (b) Western blot analysis showing that inhibition of mTORC1 by rapamycin has little effect on the upregulation of GLS downstream of oncogenic-Dbl. (c) Glutaminase activity assay using mitochondria isolated from uninduced ( + Dox) MEFs and from MEFs that had been induced for $24 \mathrm{~h}$ ( - Dox) in the absence or presence of $10 \mu \mathrm{M} \mathrm{SP600125} \mathrm{(JNK} \mathrm{inhibitor).} \mathrm{Activity} \mathrm{is} \mathrm{expressed} \mathrm{per} \mathrm{mg} \mathrm{of} \mathrm{total} \mathrm{cellular}$ protein and data presented are the mean \pm s.d. of triplicate assays. (d) RT-PCR analysis of uninduced ( + Dox) and $24 \mathrm{~h}$ induced ( - Dox $)$ MEFs $\pm 10 \mu \mathrm{M}$ SP600125, showing relative levels of the GLS transcript. The data presented are the RQ values, with error bars marking RQ max and RQ min, from triplicate reactions. (e) Western blot analysis showing that the AP-1 inhibitor SR11302 (10 $\mu \mathrm{M})$ completely blocks GLS expression in oncogenic-Dbl-induced MEFs. (f) RT-PCR analysis showing that transient transfection of uninduced (+ Dox) MEFs with constitutively activated JNK/MKK fusion constructs leads to increased GLS mRNA abundance. The data presented are the RQ values, with error bars marking RQ max and RQ min, from triplicate reactions. (g) Western blot analysis of the samples from the previous panel, showing that ectopic expression of constitutively activated JNK fusion constructs in uninduced MEFs leads to increased phosphorylation of c-Jun and upregulated GLS protein levels. (h) RT-PCR analysis showing that transient transfection of uninduced ( + Dox) MEFs with a construct for expressing the JUN proto-oncogene leads to increased GLS mRNA abundance. The data presented are the $\mathrm{RQ}$ values, with error bars marking RQ max and RQ min, from triplicate reactions. (i) Western blot analysis of the samples from the previous panel, showing that ectopic expression of JUN in uninduced MEFs leads to increased levels of phospho-c-Jun and upregulated GLS protein levels. Note that the ectopically expressed c-Jun contains a V5-tag, and consequently runs at a slightly higher molecular weight than endogenous c-Jun. Relative densitometry data are the mean \pm s.d. of triplicate blots. Differences were analysed with Student's $t$-test. ${ }^{\star} P<0.05,{ }^{\star \star} P<0.01$. 
inhibitor of the AP-1 family of transcription factors to which c-Jun belongs ${ }^{33}$. This resulted in the complete loss of GLS to undetectable levels (Fig. 3e).

The experiments above suggested that the signal to elevate GLS expression downstream of oncogenic-Dbl is transmitted via JNKmediated phosphorylation of a Jun-family AP-1 transcription factor, most likely c-Jun, since it is highly expressed in these cells (Fig. 2a), in contrast to JunB and JunD (Supplementary Fig. 2c). To test whether JNK and c-Jun can upregulate GLS expression, we transiently transfected uninduced MEFs with constructs for expressing constitutively activated MKK/JNK fusion proteins ${ }^{34}$ or c-Jun. Uninduced cells were cultured for $48 \mathrm{~h}$ after transfection (in $10 \%$ FBS medium, as transfection reagents harmed viability of MEFs in $0.5 \%$ FBS medium), and then analysed by RT-PCR and western blot. Ectopic expression of JNK1, JNK2 or JNK3 fusion-constructs all led to elevated GLS transcript levels relative to vector-control cells (Fig. 3f). Western blot analyses of whole-cell lysates showed that phospho-c-Jun and GLS were both elevated downstream of the JNK constructs (Fig. 3g). Similarly, ectopic expression of the JUN gene resulted in upregulated GLS transcript levels (Fig. $3 \mathrm{~h}$ ), and elevated levels of both phospho-c-Jun and GLS (Fig. 3i).

Collectively, these results indicate that, downstream of oncogenic Rho GTPase signalling, JNK transmits a signal to increase transcription of the GLS gene by phosphorylating and activating the oncogenic transcription factor c-Jun. This prompted us to investigate the possible role of c-Jun in regulating GLS expression in human cancer cells.

c-Jun and GLS correlate in human breast cancer cell lines. Because the JUN proto-oncogene is overexpressed, and associated with aggressive behaviour, in a subset of human breast cancers $^{35-37}$, we probed a panel of 12 breast cancer cell lines for c-Jun and GLS. First, we obtained an estimate of the glutamine dependence of each cell line by assaying proliferation over 6 days in culture medium containing either 2.0 or $0.1 \mathrm{mM}$ glutamine (Supplementary Fig. 3). We then collected cells at $\sim 60 \%$ confluency from complete RPMI medium (10\% FBS, $2 \mathrm{mM}$ glutamine) and probed whole-cell lysates by western blot, with samples ordered by increasing glutamine dependence of the cell line, left to right. This revealed a very strong correlation $(R=0.85)$ between relative levels of c-Jun and GLS (Fig. 4a and Supplementary Fig. 4a). In particular, cell lines with high endogenous c-Jun levels (BT-549, Hs 578T, MDA-MB-231 and TSE) all showed highly elevated GLS levels. The level of c-Jun also correlated very strongly with the glutamine dependence of the cell lines $(R=0.83$; see Supplementary Fig. $4 \mathrm{~b})$ and moderately strongly with their proliferation rates $(R=0.63$; see Supplementary Fig. 4c). The abundance of activated c-Jun, as indicated by phosphorylation at residue S73 (a JNK target residue), similarly correlated very strongly with GLS (Fig. 4a), whereas the related Jun-family transcription factors, JunB and JunD, showed less correlation (Fig. 4b). Relative levels of glutamate dehydrogenase (GLUD1/2) showed little variation among the cell lines (Supplementary Fig. 5).

The JNK1 and JNK2 proteins each exist as two splice variants, p46 (predominantly JNK1) and p54 (predominantly JNK2 $)^{30,38,39}$. Whereas $\mathrm{p} 46 \mathrm{JNK} 1$ is the primary kinase for activating c-Jun, endogenous p54 JNK2 can negatively regulate c-Jun stability ${ }^{30}$. We found that $446 \mathrm{JNK}$ is expressed and phosphorylated to a similar extent across the panel of breast cancer cell lines (Fig. 4c). In contrast, although p54 JNK is expressed in all cell lines, it only shows strong phosphorylation in ZR-75-1 and CAMA-1 (Fig. 4c). Given that p46 JNK is activated in all cell lines, and that phosphorylated c-Jun is proportional to total c-Jun across the panel (Fig. 4a), we conclude that differences in total c-Jun activity between the cell lines are due primarily to variations in c-Jun protein levels, rather than to differences in JNK activity.

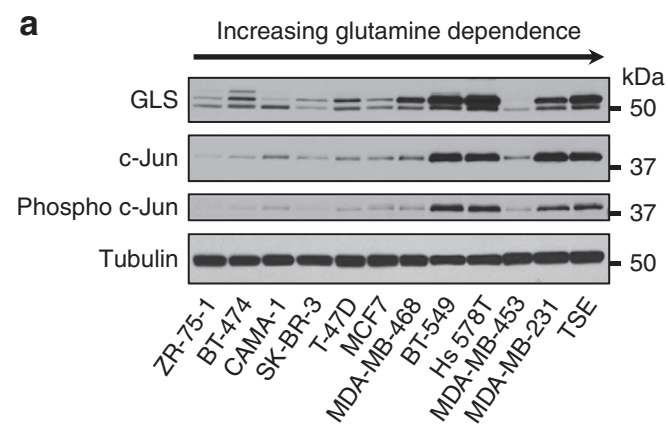

b
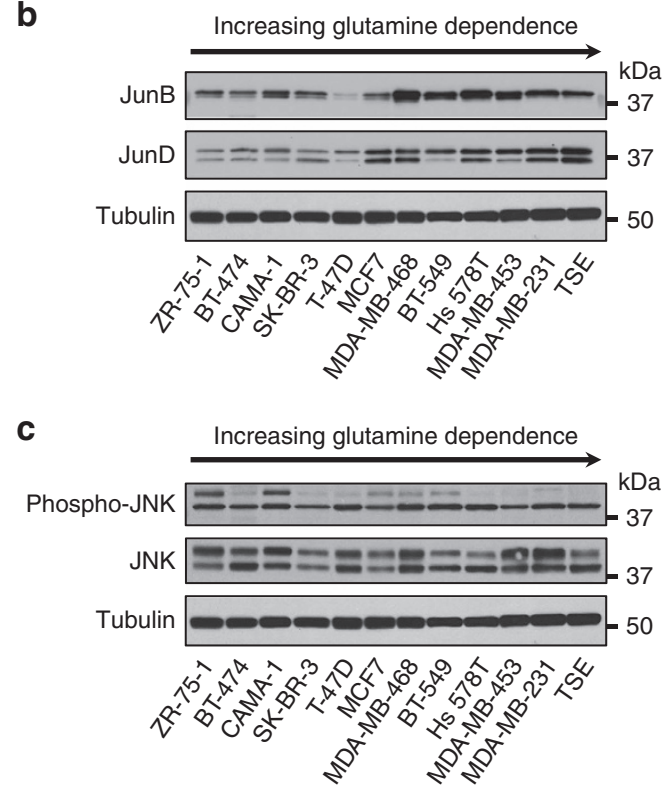

d

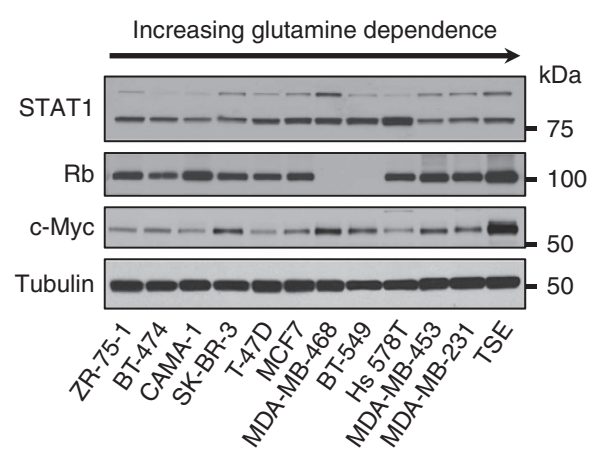

Figure 4 | c-Jun correlates strongly with GLS levels in human breast cancer cell lines. Cells were collected at $\sim 60 \%$ confluency from RPMI growth medium supplemented with $10 \%$ FBS, and whole-cell lysates prepared and analysed by western blot. Samples were ordered according to glutamine dependence, increasing from left to right (Supplementary Fig. 3). (a) Correlation between c-Jun/phospho-c-Jun and GLS levels. Quantification of GLS and c-Jun band intensities allowed a Pearson correlation coefficient of 0.85 to be determined (Supplementary Fig. 4a). (b) Other Jun-family members do not correlate strongly with GLS levels. (c) Under 10\% FBS culture conditions, p46 JNK (lower band) is active in all of the breast cancer cell lines. Neither JNK nor phospho-JNK correlate with GLS levels. (d) Other reported regulators of GLS expression do not strongly correlate with GLS levels. 
We also probed lysates for three previously reported regulators of GLS, namely c-Myc and STAT1 that upregulate GLS ${ }^{22,23,40}$, and the retinoblastoma protein $(\mathrm{Rb})$, loss of which leads to elevated GLS levels through an unknown mechanism ${ }^{41}$ (Fig. 4d). None of these proteins correlated with GLS, although we note that all three are subject to post-translational regulation.

The GLS promoter directly binds c-Jun at a consensus motif. We next examined whether c-Jun binds directly to the GLS promoter. The promoter region for the human GLS gene was analysed to position $-5,000$ bp relative to the transcription start site (TSS), and a number of putative c-Jun binding sites $(<15 \%$ dissimilarity to the consensus sequence) were identified using the $\mathrm{PROMO}^{42}$ resource (Supplementary Fig. 6). An earlier genomewide survey of c-Jun binding sites found them to be located in close proximity to the TSS, and mammalian transcription factor binding sites in general are strongly enriched around $-200 \mathrm{bp}$ from the TSS ${ }^{35,43}$. We found a close match to the consensus c-Jun binding motif (TGA[G/C]TCA) at position $-188 \mathrm{bp}$ relative to the TSS of human GLS ( $5^{\prime}$-TGACTCC- $3^{\prime}$ ) (Supplementary Figs 6 and $7 \mathrm{a}$ ). A close match to the c-Jun consensus motif is also present at position $-200 \mathrm{bp}$ in the mouse GLS promoter $\left(5^{\prime}\right.$-TGACACA- $\left.3^{\prime}\right)$ (Supplementary Fig. 7b). Exact matches to the c-Jun consensus motif $\left(5^{\prime}\right.$-TGAGTCA- $\left.3^{\prime}\right)$ were also found at position $-2,211 \mathrm{bp}$ for human GLS, and positions $-2,469$ and $-1,545$ bp for mouse GLS.

We then carried out chromatin immunoprecipitations (ChIPs) to test whether c-Jun binds directly to the GLS promoter. For these experiments, we used MDA-MB-231 breast cancer cells, which have high endogenous levels of both GLS and c-Jun (Fig. 4a). Briefly, cross-linked chromatin was digested to a length of $\sim 150-900 \mathrm{bp}$, and an antibody against endogenous c-Jun was used to immunoprecipitate c-Jun/DNA complexes. A parallel immunoprecipitation was carried out using IgG, as a negative control. Protein-DNA cross-links were then reversed, and RT-PCR was performed using primers designed to amplify a 196-bp fragment centred on the putative c-Jun binding site at position $-188 \mathrm{bp}$ relative to the TSS (Supplementary Fig. 7a). This yielded a strong signal from the c-Jun ChIP relative to the IgG ChIP, indicating that c-Jun binds to this region of the GLS promoter (Fig. 5a). Similar results were obtained using two additional sets of primers to amplify slightly shorter fragments also centred on the predicted c-Jun binding site (Supplementary Fig. 8).

c-Jun regulates GLS expression and BPTES sensitivity. Since c-Jun directly binds the promoter of the GLS gene, and endogenous levels of c-Jun and GLS correlate strongly in human breast cancer cell lines, we determined whether inhibition of JNK, inhibition of AP-1 family transcription factors, or knockdowns of c-Jun, affected GLS levels in these cells. We first inhibited JNK using $15 \mu \mathrm{M}$ SP600125 in the high-GLS cell lines MDA-MB-231 and TSE. This led to a sharp decrease in phospho-c-Jun levels, and GLS expression was strongly suppressed within $24 \mathrm{~h}$ and remained so through $72 \mathrm{~h}$ (Fig. 5b). We also directly inhibited AP-1 transcriptional activity in MDA-MB-231 cells using SR11302 (48 h), and observed a dose-dependent suppression of GLS expression (Fig. 5c). Reciprocally, ectopic expression of a constitutively activated JNK1 fusion protein in MDA-MB-231 cells, under low serum ( $0.5 \% \mathrm{FBS})$ conditions, resulted in elevated c-Jun phosphorylation and elevated GLS levels (Fig. 5d).

In BT-549 cells, which are reported to be relatively drugresistant ${ }^{44}$, treatment with $15 \mu \mathrm{M}$ SP600125 caused only a very modest inhibition of c-Jun phosphorylation (Fig. 5e, left panel). Consistent with this, SP600125 treatment did not lead to decreased GLS levels in this cell line, and we therefore used siRNAs to knockdown JUN expression. Prolonged depletion of c-Jun in BT-549 cells (and in other cell lines with high endogenous c-Jun levels) could not be tolerated and resulted in cell death. Nevertheless, whole-cell lysates from BT-549 cells treated with JUN-targeted siRNAs and collected after $48 \mathrm{~h}$ repeatedly showed that GLS levels were lower in c-Jun-depleted cells (Fig. 5e, right panel). Quantification of band intensities confirmed that the degree of c-Jun depletion correlated with the degree of GLS depletion.

The results above indicate that the relationship between c-Jun and GLS levels in breast cancer cells is not only correlative but is also causative. We therefore examined whether cell lines with high c-Jun levels are more sensitive to the GLS inhibitor BPTES. Proliferation assays were carried out for all 12 breast cancer cell lines under a range of BPTES concentrations. Defining highly sensitive cell lines as those with an $\mathrm{IC}_{50}<10 \mu \mathrm{M}$ BPTES, none of the eight cell lines with low c-Jun levels were highly sensitive to BPTES, whereas three of the four cell lines with high c-Jun levels were highly sensitive (Supplementary Table 1). The one exception was the drug-resistant cell line BT-549. Representative dose curves for a highly sensitive cell line (MDA-MB-231, $\mathrm{IC}_{50}=1.8$ $\mu \mathrm{M})$, a moderately resistant cell line $\left(\mathrm{T}-47 \mathrm{D}, \mathrm{IC}_{50}=20 \mu \mathrm{M}\right)$, and a highly resistant cell line (CAMA-1, $\left.\mathrm{IC}_{50}=\mathrm{n} / \mathrm{a}\right)$ are shown (Fig. 5f). The much greater BPTES sensitivity of the high c-Jun cell lines, Hs 578T, MDA-MB-231 and TSE, relative to the low c-Jun lines, is illustrated by the response to $2 \mu \mathrm{M}$ BPTES (Fig. $5 \mathrm{~g}$ and Supplementary Fig. 9).

Inhibition of JNK by SP600125 led to a sharp decrease in GLS levels in MDA-MB-231 (and other) breast cancer cells (see above). We therefore carried out proliferation assays to compare BPTES sensitivity of MDA-MB-231 cells cultured in the absence or presence of $15 \mu \mathrm{M}$ SP600125. Treatment with the JNK inhibitor sharply decreases the proliferation rate relative to untreated cells. However, the slow proliferation that still occurs in the presence of SP600125 is markedly desensitized to BPTES, with the $\mathrm{IC}_{50}$ for BPTES shifting $\sim 7$-fold from 1.8 to $12 \mu \mathrm{M}$ (Fig. 5h).

c-Jun is sufficient to elevate GLS in breast cancer cells. Using the MDA-MB-468 breast cancer cell line, which exhibits relatively low endogenous levels of c-Jun and moderate levels of GLS (Fig. 4a), we generated derivative cell lines that stably overexpress $J U N-V 5$ or carry the empty plasmid vector. We then collected vector-control cells and JUN-overexpressing cells at $\sim 60 \%$ confluency from RPMI medium (10\% FBS, $2 \mathrm{mM}$ glutamine), and probed whole-cell lysates for GLS, c-Jun and V5-tag by western blot (Fig. 6a). This showed that GLS levels are elevated in the $J U N$-overexpressing cells relative to the vector-control cells. Mitochondria isolated from the two cell lines were assayed for glutaminase activity, which was markedly higher in the JUN-overexpressing cells (Fig. 6b). Moreover, RT-PCR analysis confirmed that GLS transcript levels were upregulated in $J U N$-overexpressing cells relative to vector-control cells (Fig. 6c). We made equivalent derivative cell lines of the MCF7 parental cell line, which has very low endogenous levels of both c-Jun and GLS (Fig. 4a), and similarly found that GLS expression was upregulated in $J U N$-overexpressing cells (Supplementary Fig. 10a).

The level of c-Myc did not vary between the vector-control and $J U N$-overexpressing cells (Fig. 6d), and treatment with the c-Myc inhibitor 10058-F4 at concentrations up to $60 \mu \mathrm{M}(48 \mathrm{~h})$ had little effect on GLS levels in the derivative cell lines (Fig. 6e and Supplementary Fig. 10b). In contrast, treatment with the AP-1 inhibitor SR11302 $(1-10 \mu \mathrm{M})$ caused a dose-dependent 
a

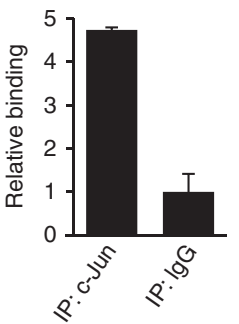

b

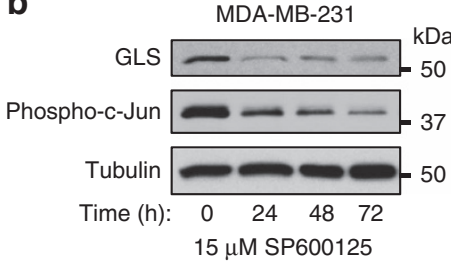

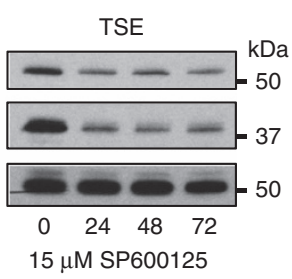

c

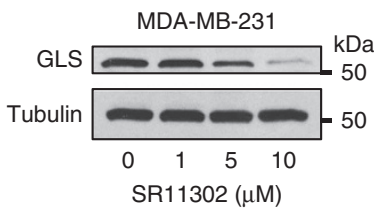

d

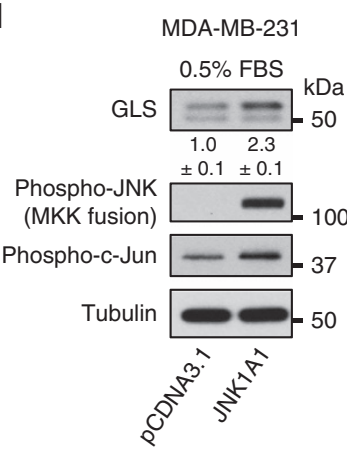

f

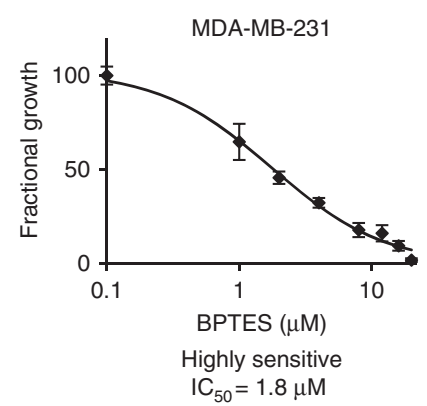

e

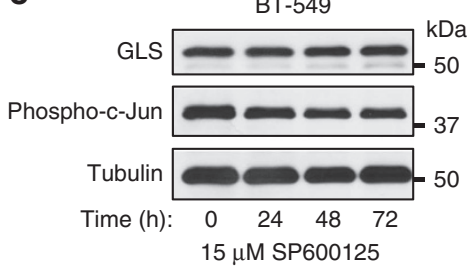

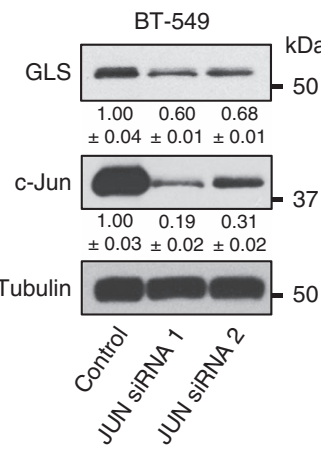

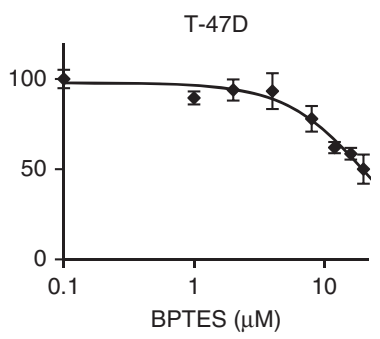

Moderately resistant $\mathrm{IC}_{50}=20.3 \mu \mathrm{M}$

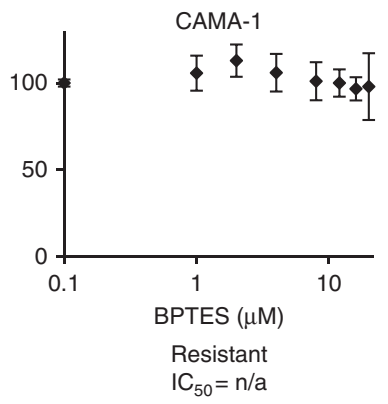

g

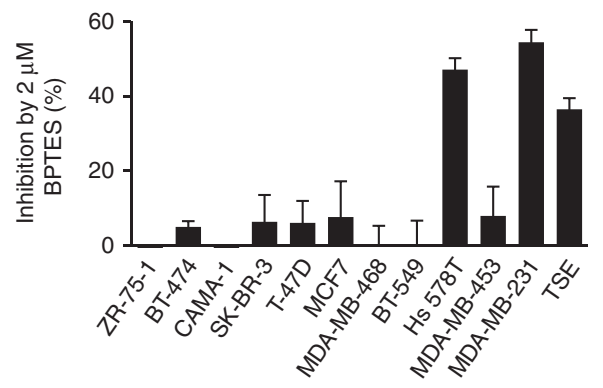

h

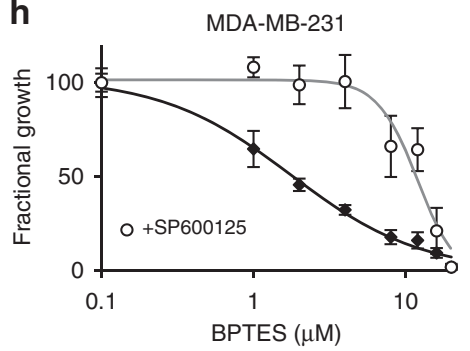

Figure 5 | Inhibition of c-Jun suppresses GLS expression and BPTES sensitivity in human breast cancer cell lines. (a) ChIP analysis showing that c-Jun binds to the GLS promoter. Complexes containing c-Jun were immunoprecipitated from cross-linked, digested, chromatin isolated from MDA-MB-231 cells. A parallel immunoprecipitation using rabbit lgG was carried out as a negative control. Following reversal of cross-links and purification of DNA, RT-PCR was run using primers designed to amplify a 196-bp fragment centred on the putative c-Jun binding site at position - 188 bp relative to the TSS. The data presented are the RQ values, with error bars marking RQ max and RQ min, from triplicate reactions. (b) Western blot analysis showing that treatment of MDA-MB-231 or TSE cells with the JNK inhibitor SP600125 (15 $\mu \mathrm{M})$ leads to decreased phosphorylation of c-Jun, and decreased GLS levels. (c) Treatment of MDA-MB-231 cells with the AP-1 inhibitor SR11302 (1-10 $\mu \mathrm{M}$ ) for $48 \mathrm{~h}$ results in a dose-dependent decrease in GLS. (d) Western blot analysis showing that transient transfection of MDA-MB-231 cells with a constitutively activated JNK fusion construct results in increased c-Jun phosphorylation and upregulated GLS levels. Cells were collected $48 \mathrm{~h}$ after transfection. (e) Western blot analysis showing that in the drug-resistant breast cancer cell line BT-549, treatment with $15 \mu \mathrm{M}$ SP600125 has little effect on c-Jun phosphorylation and does not lead to decreased GLS levels (left panels). However, knockdown of JUN expression using siRNAs leads to decreased GLS levels. Relative band intensities are indicated. (f) Representative BPTES dose curves showing the effect of BPTES on the proliferation of breast cancer cell lines over 6 days. Curves were fitted using SigmaPlot, with data from triplicate assays. (g) Sensitivity of breast cancer cell lines to GLS inhibition, as indicated by inhibition of proliferation over 6 days by $2 \mu$ M BPTES. Of the high-c-Jun lines, only the drug-resistant BT-549 cells were not highly sensitive to BPTES. None of the low-c-Jun lines were highly sensitive. Data presented are the mean $\pm \mathrm{s}$.d. of triplicate assays. (h) BPTES dose curves for MDA-MB-231 cells $\pm 15 \mu \mathrm{M}$ SP600125, showing that inhibition of JNK desensitizes cells to GLS inhibition (the $\mathrm{IC}_{50}$ for BPTES shifts from 1.8 to $12 \mu \mathrm{M}$ ). Curves were fitted using SigmaPlot, with data from triplicate assays. Relative densitometry data are the mean \pm s.d. of triplicate blots. 
a

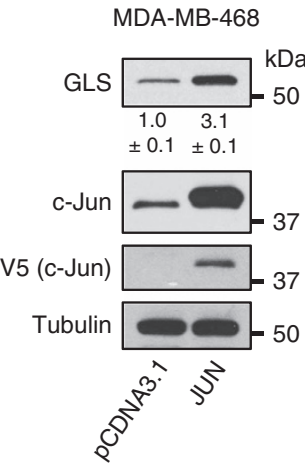

d

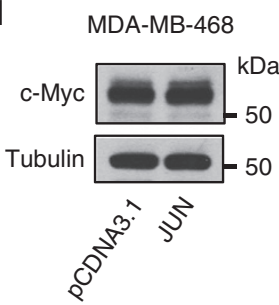

b

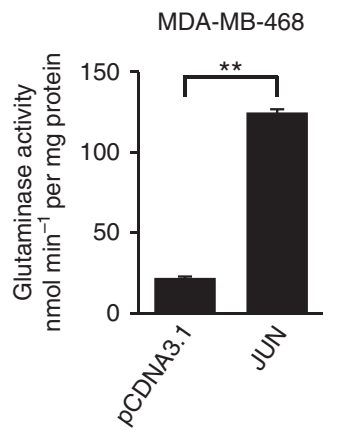

e

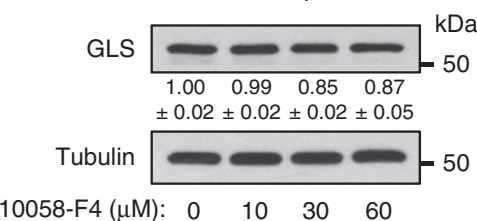

10058-F4 (uM): $0 \quad 10 \quad 30 \quad 60$

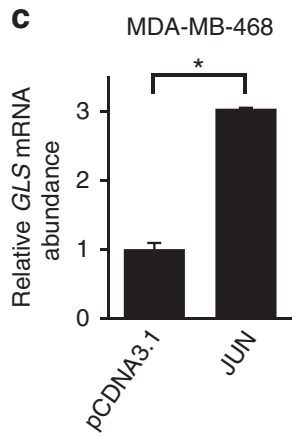

f

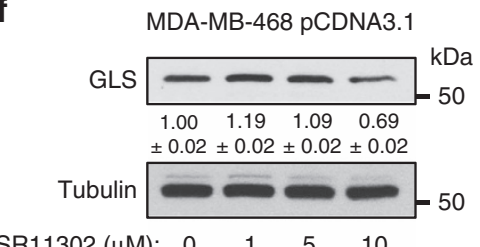

SR11302 ( $\mu \mathrm{M}): 0 \quad 0 \quad 1 \quad 5 \quad 10$
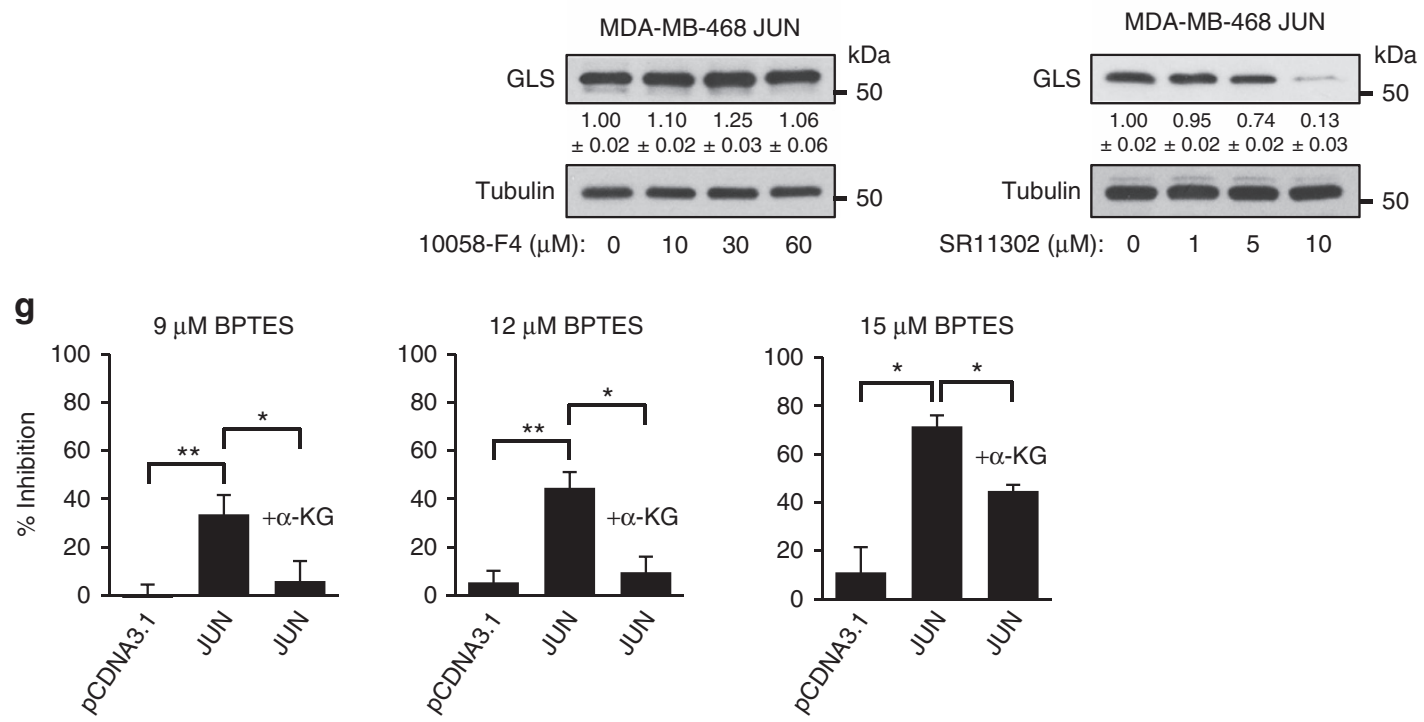

Figure 6 | c-Jun increases GLS expression and BPTES sensitivity in breast cancer cells. (a) Western blot analysis of GLS and c-Jun levels in whole-cell lysates of MDA-MB-468 cells, stably carrying either pCDNA3.1 or the JUN-V5 expression vector pCDNA3.1-JUN. Relative band intensities are indicated for GLS. (b) Glutaminase activity assay using mitochondria isolated from the derivative MDA-MB-468 cell lines. Activity is expressed per mg of total cellular protein, and data presented are the mean \pm s.d. of triplicate assays. (c) RT-PCR analysis of the derivative MDA-MB-468 cell lines, showing relative abundance of the GLS transcript. The data presented are the RQ values, with error bars marking RQ max and RQ min, from triplicate reactions. (d) Western blot analysis showing c-Myc levels in the derivative cell lines. (e) Western blot analysis showing that treatment of vector-control, or JUN-overexpressing, MDA-MB-468 cells with the c-Myc inhibitor 10058-F4 at concentrations up to $60 \mu \mathrm{M}$ for $48 \mathrm{~h}$ has little effect on GLS levels. Relative band intensities are indicated for GLS. (f) Western blot analysis showing that treatment with the AP-1 inhibitor SR11302 (1-10 $\mu \mathrm{M})$ for $48 \mathrm{~h}$ leads to a dose-dependent decrease in GLS levels. The effect is especially pronounced in JUN-overexpressing cells. (g) Cell proliferation assays for MDA-MB-468 cells stably carrying pCDNA3.1 or the JUN expression vector. Cells were seeded in 12-well dishes at a density of $1 \times 10^{4}$ cells per well, cultured for 6 days in the absence or presence of $9 \mu \mathrm{M}$ (left panel), $12 \mu \mathrm{M}$ (middle panel) or $15 \mu \mathrm{M}$ (right panel) BPTES $\pm 1 \mathrm{mM}$ dimethyl $\alpha-K G$. Inhibition of proliferation by BPTES treatment is plotted as a percentage. The data presented are the mean \pm s.d. of triplicate assays. Relative densitometry data are the mean \pm s.d. of triplicate blots. Differences were analysed with Student's $t$-test. ${ }^{\star} P<0.05,{ }^{* \star} P<0.01$.

suppression of GLS in both cell lines, an effect that was especially pronounced in the JUN-overexpressing cells (Fig. 6f).

Overexpression of JUN sensitizes cancer cells to BPTES. As described above, MDA-MB-231 cells treated with the JNK inhibitor SP600125 become desensitized to the GLS inhibitor BPTES (Fig. 5h). To complement this result, we carried out proliferation assays to determine whether overexpression of the JUN proto-oncogene is sufficient to increase the BPTES sensitivity of MDA-MB-468 cells. Although vector-control cells showed some response to BPTES treatment, overexpression of JUN greatly sensitized cells to BPTES treatment (Fig. 6g). Supplementation of the medium with $1 \mathrm{mM}$ dimethyl $\alpha$-KG completely rescued cells from $\leq 12 \mu \mathrm{M}$ BPTES, and partially rescued cells from higher BPTES concentrations (Fig. 6g). Although the parental and vector-control MDA-MB-468 cell lines 
showed little dependence on the GLS reaction, both are moderately glutamine-dependent (Supplementary Figs 3 and 11). This reflects the importance of an exogenous glutamine supply for other metabolic processes that do not involve the GLS reaction, such as nucleotide biosynthesis and protein synthesis. Glutamine dependence of the JUN-overexpressing derivative cell line was unchanged from that of the parental cells (Supplementary Fig. 11).

c-Jun regulated transcript levels in invasive breast cancer. To establish further the relationship between c-Jun activity and GLS expression in breast cancer, we used the cBioportal $^{45,46}$ suite of tools (www.cbioportal.org) to analyse data from The Cancer Genome Atlas (TCGA) Breast Invasive Carcinoma (TCGA, provisional) data set (959 samples). In many cancers, upregulation of c-Jun occurs at the post-transcriptional and post-translational levels, but not at the transcriptional level (see Discussion). Consequently, JUN mRNA levels are rarely upregulated, even though c-Jun protein levels can be highly elevated. Consistent with these findings, TCGA data showed that JUN mRNA was elevated ( $z$ score $>2.0$ ) in only $3 \%$ of invasive breast carcinoma samples. We therefore determined whether the expression levels of some validated c-Jun transcriptional targets correlate with those of GLS. We selected only confirmed direct targets of c-Jun (and not v-Jun) that contain a c-Jun binding site within the promoter region ${ }^{35,47-49}$, and we eliminated from the list those c-Jun target genes that are frequently altered at the genomic level within the data set (for example, CCND1, amplified in $16 \%$ of samples, and TP53, mutated in $31 \%$ of samples). Using the resulting list of c-Jun targets (Supplementary Table 2), we found that transcripts that are upregulated by c-Jun almost invariably correlate positively with GLS mRNA levels, whereas those targets that are transcriptionally repressed by c-Jun exhibit a negative correlation with the GLS transcript (Fig. 7; see also Supplementary Figs 12 and 13).

\section{Discussion}

In proliferating cells, the TCA cycle serves as a major source of biosynthetic precursors. Metabolic intermediates that are lost to

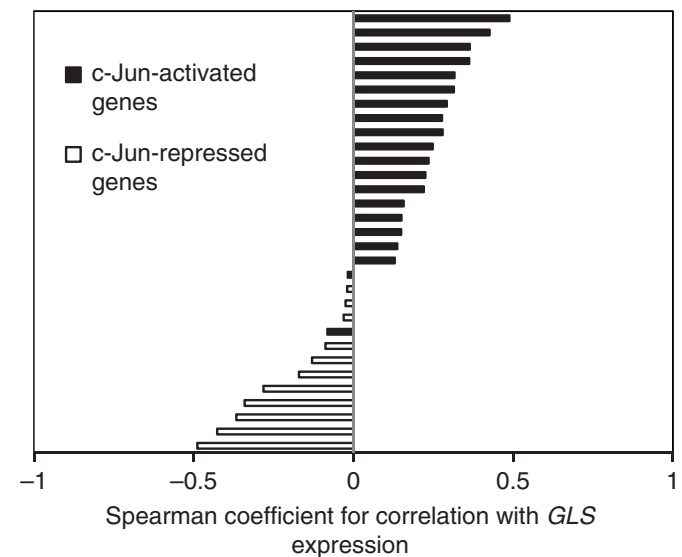

Figure 7 | The GLS transcript correlates with established c-Jun target transcripts in invasive breast cancer. Bar chart showing the Spearman correlation coefficients between mRNA levels of GLS and of established c-Jun transcriptional targets. Transcripts that are upregulated by c-Jun are shown in black, and almost exclusively correlate positively with GLS.

Transcripts that are repressed by c-Jun are shown in white, and all correlate negatively with GLS. Correlation plots were prepared and correlation coefficients determined using the cBioportal suite of tools and data from The Cancer Genome Atlas (TCGA) Breast Invasive Carcinoma (TCGA, provisional) data set. anabolic pathways must be rapidly replenished, and the most abundant carbon sources for these anaplerotic reactions are glucose and glutamine. Indeed, anaplerosis is a key function underlying the upregulated consumption of these nutrients by cancer cells ${ }^{10,11,50}$. An important route for the delivery of glutamine-derived carbon into the TCA cycle begins with the hydrolysis of glutamine to glutamate, a reaction catalysed by glutaminase. The TCA cycle intermediate $\alpha$-ketoglutarate can then be generated through the action of glutamate dehydrogenase, or through transaminase activities.

In this study, we used an inducible MEF system for hyperactivating Rho GTPase signalling, which we previously linked to increased mitochondrial GLS activity in NIH-3T3 cells ${ }^{20}$, and also to upregulated glutamine-mediated anaplerosis for oxidative TCA cycle flux in $\mathrm{MEFs}^{51}$. Ectopic expression of oncogenic-Dbl in NIH-3T3 cells leads to elevated levels of the GLS transcript (Supplementary Fig. 14a), but the lower background level of GLS in MEFs relative to NIH-3T3 cells (Supplementary Fig. 14b) allows for a much clearer read-out, by RT-PCR and especially by western blot, of changes in GLS expression. By selectively inhibiting signalling pathways activated downstream of the Rho GTPases in the inducible system, we have now discovered that the c-Jun N-terminal kinase (JNK) and the oncogenic transcription factor c-Jun are critical for upregulating GLS at the transcript and protein levels. We also found that in human breast cancer cell lines, c-Jun levels correlate strongly with GLS levels and with sensitivity to the GLS inhibitor BPTES. Moreover, we determined that c-Jun directly binds the promoter of the GLS gene and increases its expression. Importantly, this not only leads to elevated mitochondrial glutaminase activity, but also causes cancer cells to become more dependent on glutamine-mediated anaplerosis.

The c-Jun transcription factor is the cellular homologue of $\mathrm{v}$-Jun, the transforming oncoprotein of avian sarcoma virus 17 (refs 52,53). A key role for c-Jun in both healthy and neoplastic tissue is to drive cell cycle progression, and fibroblasts derived from JUN-null mouse embryos exhibit a severe defect in proliferation ${ }^{54}$. The c-Jun protein is phosphorylated at the M/G1 transition ${ }^{55}$, and loss of c-Jun or expression of mutants defective for JNK-catalysed phosphorylation leads to a G2/M cell cycle block ${ }^{56}$. One transcriptional target of c-Jun is the CCND1 gene, which encodes the $\mathrm{G} 1$ to $\mathrm{S}$ phase regulator cyclin D1 (ref. 57). c-Jun also binds to a variant AP-1 site in the TP53 gene promoter, leading to suppression of p53 and of the p53-regulated cyclin-dependent kinase inhibitor p21 (ref. 54). The net effect is that c-Jun increases the activity of G1 cyclin-dependent kinase complexes.

When taken together, these findings provide a biological rationale for the positive regulation of GLS by c-Jun that we describe. In order for sustained proliferation to occur, signalling to the cell cycle machinery must be coordinated with reprogramming of cellular metabolism to support biomass accumulation. By simultaneously driving cell cycle progression and upregulating GLS expression, c-Jun promotes cell proliferation and also activates TCA cycle anaplerosis to replenish metabolites that have been directed to biosynthetic pathways (Fig. 8). Notably, two other reported regulators of GLS expression are involved in cell cycle control. The Rb protein prevents G1 to $S$ phase progression ${ }^{58}$, and loss of this tumour suppressor leads to upregulation of $\mathrm{GLS}^{41}$. Similarly, the oncogenic transcription factor c-Myc promotes G1 to $S$ phase transition, and is a positive regulator of GLS expression ${ }^{22,23}$.

Mirroring the ability of v-Jun to cause avian sarcoma, the JUN gene is highly amplified and overexpressed in aggressive human sarcomas ${ }^{59}$, and in an analysis of copy number alterations across 3,131 diverse cancer samples, JUN was found to be significantly 


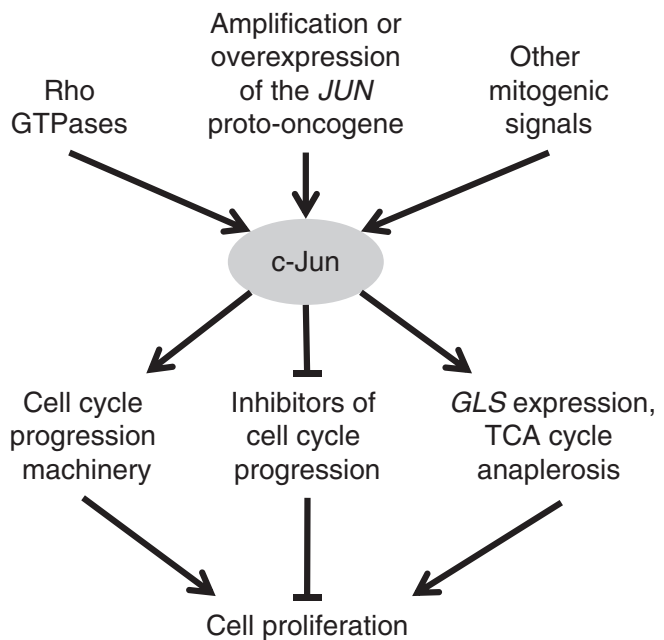

Figure 8 | c-Jun coordinates cell cycle progression with metabolic reprogramming. Diagram summarizes the coordinated regulation of cell proliferation by c-Jun. Transcriptional activity of c-Jun can be enhanced by activating phosphorylations downstream of mitogenic signalling pathways, including those mediated by the Rho family of GTPases. c-Jun can also be upregulated by amplification or increased expression of the JUN proto-oncogene. A key role for c-Jun is to drive cell cycle progression, which is achieved through transcriptional repression of cell cycle inhibitors and transcriptional activation of cell cycle progression machinery. In this study, we report that c-Jun also controls metabolic reprogramming to support proliferation, by upregulating GLS expression and thereby stimulating delivery of glutamine-derived carbon into the TCA cycle.

amplified across the entire data set ${ }^{60}$. In many cancers, protein levels of c-Jun are highly upregulated even in the absence of JUN amplifications or elevated transcription of the JUN gene. This can be due to both translational activation and increased protein stability. For example, c-Jun is highly elevated in melanoma cells as a result of increased translation following loss of mi-R125b, which binds to the coding region of the JUN transcript and suppresses translation in normal melanocytes ${ }^{61}$. In breast cancer cells, c-Jun is also upregulated at the protein level but not at the transcriptional level ${ }^{37}$. One mechanism for this involves downregulation of the tumour suppressor COP1 E3 ubiquitin ligase, which targets c-Jun for degradation ${ }^{37}$. Indeed, among the cell lines used in the present study, BT-549, Hs 578T and MDAMB-231 are reported to have low COP1 levels, whereas MCF7 and T47D have elevated COP1 (ref. 37).

For transcriptional activity, c-Jun and related transcription factors must form a dimeric complex, which is known as AP-1 and consists of Jun-Jun or Jun-Fos dimers. Jun-family members (c-Jun, JunB and JunD) can dimerize with themselves or with Fos proteins, whereas Fos-family members (c-Fos, Fra-1, Fra-2 and FosB) must heterodimerize with a Jun protein ${ }^{57}$. This raises some questions concerning the fine-tuning of GLS regulation. Of the AP-1 transcription factors, c-Jun is the most potent transcriptional activator. However, Jun-Fos heterodimers are more stable and efficient at driving transcriptional activation than Jun-Jun dimers ${ }^{57}$. Importantly, the promoter specificity of dimers containing c-Jun differs according to the dimerization partner, and the relative abundances of potential partners vary between cell types ${ }^{62,63}$. Consequently, the Jun- and Fos-family members that are expressed within a given cell likely influence the regulation of GLS expression by c-Jun, and it is possible that JunB or JunD might substitute for c-Jun in some contexts.

High levels of c-Jun are associated with aggressive, invasive and metastatic behaviour in breast cancer. Ectopic overexpression of
JUN in the MCF7 cell line leads to increased invasion and tumorigenicity, whereas a dominant-negative variant of c-Jun causes cell cycle arrest ${ }^{64,65}$. Several studies have found that $c$-Jun is specifically elevated in triple-negative breast cancer (TNBC) cells $^{35,37,66}$ and $M Y C$ expression is also reported to be upregulated in $\mathrm{TNBC}^{67}$. This could explain why TNBC cell lines have higher GLS levels and greater sensitivity to the GLS inhibitor CB-839 (a BPTES derivative) than receptor-positive breast cancer cells ${ }^{21}$.

In conclusion, we have identified a JNK/c-Jun-dependent signalling pathway that is responsible for upregulating GLS levels during cellular transformation. Moreover, we show that the c-Jun transcription factor is an important regulator of GLS expression in breast cancer, and can drive cellular dependence on the glutaminase reaction, thus conferring sensitivity to the GLS inhibitor BPTES. Our findings link a key player in cell cycle progression with a metabolic pathway that supports cell proliferation, and illustrate that re-programming of cellular metabolism is intricately connected with oncogenic transformation. Furthermore, our work reveals a novel function for the c-Jun oncoprotein in cancer, and suggests why certain types of cancer such as triple-negative breast cancer might be more susceptible to glutaminase-targeted therapy.

\section{Methods}

Isogenic inducible MEF system for oncogenic-Dbl expression. The inducible MEF line used in this study was generated as described previously ${ }^{51}$. Briefly, the gene encoding oncogenic-Dbl was sub-cloned into vector pTRE-HA (Clontech). Parental MEFs containing the transcriptional transactivator tTA (Clontech) were then co-transfected with the resulting pTRE-HA-onco-Dbl vector along with vector pMET-puro, in a 20:1 ratio. Following puromycin selection $(\sim 3$ weeks $)$, colonies were screened for inducible expression of oncogenic-Dbl. Cells were maintained at $37^{\circ} \mathrm{C}, 5 \% \mathrm{CO}_{2}$ atmosphere, in DMEM medium containing $4 \mathrm{mM}$ glutamine (Gibco) and supplemented with $10 \%(\mathrm{v} / \mathrm{v})$ tetracycline-free FBS (Gibco) and $0.6 \mu \mathrm{g} \mathrm{ml}^{-1}$ doxycycline. Expression of oncogenic-Dbl was induced by re-plating cells in doxycycline-free medium (10\% FBS). Residual doxycycline was removed by replacing the medium (with the appropriate concentration of FBS, and small-molecule inhibitor where relevant) after $5 \mathrm{~h}$. Uninduced control samples were treated in the same way: re-plating followed by media change at $5 \mathrm{~h}$, but with $0.6 \mu \mathrm{g} \mathrm{ml}^{-1}$ doxycycline present at all times. For glutamine-withdrawal experiments, glutamine-free DMEM medium (Gibco) was supplemented with dialysed FBS (Gibco). Growth medium containing other concentrations of glutamine was prepared by mixing appropriate volumes of DMEM ( $4 \mathrm{mM}$ glutamine) and glutamine-free DMEM.

Breast cancer cell culture and media. Breast cancer cell lines ZR-75-1, BT-474, CAMA-1, SK-BR-3, T-47D, MCF7, MDA-MB-468, BT-549, Hs 578T, MDA-MB-453 and MDA-MB-231 were obtained from the American Type Cell Culture Collection (ATCC). The TSE breast cancer cell line was kindly supplied by Dr Steven Abcouwer (University of Michigan). All breast cancer cell lines were maintained at $37^{\circ} \mathrm{C}, 5 \% \mathrm{CO}_{2}$ atmosphere, in RPMI 1640 medium containing $2 \mathrm{mM}$ glutamine (Gibco) and supplemented with 10\% FBS (Gibco). For glutamine-withdrawal experiments, glutamine-free RPMI 1640 medium (Gibco) supplemented with $10 \%$ dialysed FBS (Gibco) was used. Cell lines were periodically tested for Mycoplasma contamination.

DNA constructs. DNA primers were synthesized by Integrated DNA Technologies. Plasmid pCDNA3.1/V5-His TOPO was purchased from Life Technologies. The JUN gene was PCR amplified from plasmid pMIEG3-c-Jun, which was a gift from Alexander Dent (Addgene plasmid \# 40348) ${ }^{68}$, using primers JUN_AMP_F (5'-TTATGGATCCATGACTGCAAAGATGGAAACGACC- $\left.3^{\prime}\right)$ and JUN_AMP_R (5'-TTATGATATCAAATGTTTGCAACTGCTGCGTTAGC- $\left.3^{\prime}\right)$, which added a BamH1 site and EcoRV site, respectively. The PCR product was purified (QIAquick PCR purification kit, QIAGEN), digested with BamH1 and EcoRV (New England Biolabs), re-purified and ligated (T4 DNA ligase, New England Biolabs) into pre-digested and purified pCDNA3.1/V5-His TOPO to yield an expression vector for JUN-V5, pCDNA3.1-JUN. The construct was verified by sequencing. The plasmids pCDNA3 Flag MKK7B2Jnk1a1 (Addgene plasmid \# $19726)^{34}$, pCDNA3 Flag MKK7B2Jnk2a2 (Addgene plasmid \# 19727) ${ }^{34}$ and pCDNA3 Flag MKK7B2Jnk3a2 (Addgene plasmid \# 19729) ${ }^{34}$ were all gifts from Roger Davis. 
Antibodies and reagents. Antibodies recognizing the following proteins were purchased from Cell Signaling Technology: HA-tag (cat. no. 3724) used at 1:5,000, $\alpha / \beta$-tubulin (cat. no. 2148) used at 1:5,000, phospho-SEK1/MKK4 (S257) (cat. no. 4514) used at 1:2,000, phospho-MKK7 (S271/T275) (cat. no. 4171) used at 1:2,000, phospho-JNK (T183/Y185) (cat. no. 4668) used at 1:2,000, phospho-c-Jun (S73) (cat. no. 3270) used at 1:2,000, c-Jun (cat. no. 9165) used at 1:2,000, JunB (cat. no. 3753) used at 1:2,000, JunD (cat. no. 5000) used at 1:2,000, phospho-p38 MAPK (T180/Y182) (cat. no. 4511) used at 1:2,000, p38 MAPK (cat. no. 8690) used at 1:2,000, phospho-ERK1/2 (T202/Y204) (cat. no. 4370) used at 1:2,000, ERK1/2 (cat. no. 9102) used at 1:2,000, phospho-myosin light chain 2 (S19) (cat. no. 3675) used at 1:1,000, myosin light chain 2 (cat. no. 3672) used at 1:1,000, phospho-AMPK $\alpha$ (T172) (cat. no. 4188) used at 1:2,000, AMPK $\alpha$ (cat. no. 2603) used at 1:2,000, phospho-Akt (T308) (cat. no. 9275) used at 1:2,000, Akt (cat. no. 9272) used at 1:2,000, phospho-p70 S6 kinase (T389) (cat. no. 9205) used at 1:2,000, p70 S6 kinase (cat. no. 9202) used at 1:2,000, STAT1 (cat. no. 9172) used at 1:2,000, $\mathrm{Rb}$ (cat. no. 9309) used at 1:2,000, Glut1 (cat. no. 12939) used at 1:4,000, and c-Myc (cat. no. 5605) used at 1:2,000. The antibody recognizing GLS was purchased from Abgent (cat. no. AP8809b) and used at 1:8,000. Clarification of the bands recognized by this antibody is shown in Supplementary Fig. 15. Antibodies recognizing MKK4 (MEK4) (cat. no. ab33912), MKK7 (MEK7) (cat. no. ab52618) and phospho-c-Myc (T58 + S62) (cat. no. ab10568), all used at 1:4,000, were purchased from Abcam. Antibodies recognizing JNK (cat. no. sc-571) and GLUD1/2 (cat. no. sc-160383), both used at 1:2,000, were purchased from Santa Cruz Biotechnology. The antibody recognizing V5-tag was purchased from Life Technologies (cat. no. R960) and used at 1:5,000. Secondary antibodies used, as appropriate, were Cell Signaling Technology anti-rabbit IgG, HRP-linked (cat. no. 7074) or anti-mouse IgG, HRP-linked (cat. no. 7076), or Santa Cruz Biotechnology donkey anti-goat IgG-HRP (cat. no. sc-2020). BPTES was synthesized and kindly provided by Dr Scott Ulrich, Ithaca College. ROCK inhibitor Y-27632 (10 mM solution in DMSO), the JNK inhibitor SP600125 (50 mM solution in DMSO) and G418 were purchased from Calbiochem. All other reagents were purchased from Sigma-Aldrich unless otherwise stated.

Western blot analysis. Whole-cell lysates were prepared in lysis buffer $(50 \mathrm{mM}$ HEPES pH 8.0, $150 \mathrm{mM} \mathrm{NaCl}, 1 \mathrm{mM} \mathrm{Na}_{3} \mathrm{VO}_{4}, 25 \mathrm{mM} \mathrm{NaF}, 1 \%$ (v/v) Triton X-100, $1 \mathrm{mM} \mathrm{MgCl}_{2}, 50 \mathrm{mM} \beta$-glycerophosphate, $30 \mu \mathrm{g} \mathrm{ml}^{-1}$ leupeptin, $5 \mu \mathrm{g} \mathrm{ml}^{-1}$ aprotinin) and insoluble debris was pelleted by centrifugation and removed. Protein concentration was determined by Bradford assay (Bio-Rad), and lysate proteins denatured by boiling for $5 \mathrm{~min}$ in reducing SDS-sample buffer. Lysate proteins ( $20 \mu \mathrm{g}$ total protein/lane) were then resolved on Novex $4-20 \%$ Tris-glycine mini or midi protein gels (Life Technologies), and transferred to polyvinylidene difluoride membranes (PerkinElmer). Membranes were blocked in 7\% bovine serum albumin in tris-buffered saline and tween 20 (TBST) for $1 \mathrm{~h}$ at room temperature, and probed overnight at $4{ }^{\circ} \mathrm{C}$ in primary antibody solution (manufacturer recommended concentration) in TBST. They were then washed in TBST, and incubated in TBST solution containing $25 \%(\mathrm{v} / \mathrm{v})$ non-fat dry milk powder and appropriate secondary antibody at the manufacturer's recommended concentration for $1 \mathrm{~h}$. Finally, the membranes were washed in TBST, and bands imaged using Western Lightning Plus-ECL (PerkinElmer) and HyBlot ES autoradiography film (Denville Scientific Inc.).

MEF saturation density analysis. DMEM medium supplemented with $10 \%$ FBS $\pm 0.6 \mu \mathrm{g} \mathrm{ml}^{-1}$ doxycycline was added to $60 \mathrm{~mm}$ dishes ( $3 \mathrm{ml}$ per dish), and dishes were then seeded with $1 \times 10^{5} \mathrm{MEFs}$ per dish. Following cell attachment $(5 \mathrm{~h})$, growth medium was replaced with fresh medium containing appropriate supplements. Growth medium was subsequently replaced at $48 \mathrm{~h}$ intervals. At day 8 , growth medium was removed and $1.5 \mathrm{ml} 3.7 \%$ formaldehyde solution in $\mathrm{H}_{2} \mathrm{O}$ was added for $30 \mathrm{~min}$. The formaldehyde solution was removed, and $1.5 \mathrm{ml}$ crystal violet solution was added for $20 \mathrm{~min}$ at room temperature. This was then removed, and dishes washed four times with $3 \mathrm{ml} \mathrm{H}_{2} \mathrm{O}$ and allowed to dry before imaging.

MEF anchorage-independent growth assay. Uninduced or oncogenic-Dblinduced MEFs were seeded at a density of 8,000 cells per $\mathrm{ml}$ in medium containing $0.3 \%(\mathrm{w} / \mathrm{v})$ agarose, onto underlays of medium containing $0.6 \%$ agarose, in six-well plates. Both layers of medium were supplemented with appropriate concentrations of doxycycline (uninduced cells) and BPTES. Cultures were fed every 3 days, and the total number of colonies was counted after 15 days.

Cell proliferation assays. Culture medium supplemented with glutamine or with inhibitors at the described concentrations was added to 12 -well plates $(1 \mathrm{ml}$ per well), and wells were seeded with cells at day 0 as follows (numbers optimized to avoid over-confluence during the assay). TSE cells: $5 \times 10^{3}$ cells per well. MDA-MB-231, MDA-MB-453, MDA-MB-468, BT-549: $1 \times 10^{4}$ cells per well. Hs 578T, MCF7, T-47D, CAMA-1, SK-BR-3, BT-474: $1.5 \times 10^{4}$ cells per well. ZR-75-1: $2 \times 10^{4}$ cells per well. Following cell attachment, growth medium was replaced at $12 \mathrm{~h}$ and subsequently every $48 \mathrm{~h}$. At day 6, cells were trypsinized, suspended in an appropriate volume of medium, and a hemacytometer was used to determine the total number of cells per well. All cell proliferation assays were carried out in triplicate, and the mean and s.d. calculated.
Mitochondrial isolation. Mitochondria were isolated using the Qproteome mitochondria isolation kit (QIAGEN), following the manufacturer's instructions. For MEFs grown in low-serum media, $3 \times 15 \mathrm{~cm}$ dishes of cells at $\sim 70 \%$ confluency were collected, and for MDA-MB-468 cells, $2 \times 15 \mathrm{~cm}$ dishes of cells at $\sim 70 \%$ confluency were collected. The total protein of the starting material (collected cells) and of the isolated mitochondria suspension was measured by Bradford assay (Bio-Rad). This allowed glutaminase activity to be determined per unit of total cellular protein (see below).

Mitochondrial glutaminase activity assay. A two-step protocol was used to assay glutaminase activity, as previously described ${ }^{28}$. In the first reaction, glutaminase catalyses the hydrolysis of glutamine to glutamate, and in the second reaction glutamate dehydrogenase catalyses the oxidative deamination of glutamate to form $\alpha$-ketoglutarate and NADH, which can be measured through its absorbance at $340 \mathrm{~nm}$. Briefly, mitochondria (10 $\mu \mathrm{g}$ total protein) were added to $105 \mu \mathrm{l}$ of reaction mix 1 ( $20 \mathrm{mM}$ glutamine, $0.2 \mathrm{mM}$ EDTA, $50 \mathrm{mM}$ Tris-acetate, $\mathrm{pH}$ 8.6). Samples were rotated at $37^{\circ} \mathrm{C}$ for $45 \mathrm{~min}$. The reaction was then quenched by adding $10 \mu \mathrm{l}$ of $3 \mathrm{M} \mathrm{HCl}$, and samples were placed on ice. Next, $20 \mu \mathrm{l}$ of reaction mix 1 was added to $200 \mu \mathrm{l}$ of reaction mix 2 (1 unit bovine liver glutamate dehydrogenase (Sigma-Aldrich), $80 \mathrm{mM}$ Tris- $\mathrm{HCl} \mathrm{pH} 9.4,200 \mathrm{mM}$ hydrazine, $0.25 \mathrm{mM} \mathrm{ADP}$, $2 \mathrm{mM} \mathrm{NAD}$ ). The samples were mixed and incubated for $1 \mathrm{~h}$ at room temperature. The $\mathrm{A}_{340}$ was then determined against a blank in which a heat-inactivated mitochondrial sample was added to reaction mix 1. A standard curve was also prepared by adding known amounts of glutamate to reaction 2 . This allowed the amount of glutamate generated during reaction 1 to be determined. All assays were carried out in triplicate, and the mean and s.d. calculated.

Real-time PCR analysis. Total RNA was extracted from cells using the RNeasy mini kit (QIAGEN), and cDNAs were prepared using the SuperScript III firststrand synthesis system (Life Technologies). RT-PCR was carried out using the 7,500 fast real-time PCR system (Applied Biosystems), with cDNA as a template and GLS primers GLS-F (5'-TGTCACGATCTTGTTTCTCTGTG-3') and GLS-R (5'-TCATAGTCCAATGGTCCAAAG- $\left.3^{\prime}\right)$. Primers for mouse GAPDH (mGAPDH-F, $5^{\prime}$-ACAGTCCATGCCATCACTGCC- $3^{\prime}$ and mGAPDH-R, $5^{\prime}$-GCC TGCTTCACCACCTTCTTG- $3^{\prime}$ ) or human ACTB (hACTB-F, 5' -CATCGAGCA CGGCATCGTCA- $3^{\prime}$ and hACTB-R, $5^{\prime}$-TAGCACAGCCTGGATAGCAAC- $3^{\prime}$ ) were used as endogenous controls, as appropriate. All reactions were carried out using POWER SYBR green PCR master mix (Life Technologies). At least three replicates of each PCR were carried out.

Chromatin immunoprecipitation. Chromatin immunoprecipitations were performed using the SimpleChIP enzymatic chromatin IP kit (Cell Signaling Technology), following the manufacturer's instructions. MDA-MB-231 cells $(5 \times 15 \mathrm{~cm}$ dishes at $\sim 85 \%$ confluency) were used as the source of chromatin. Analysis following chromatin digestion showed that DNA was digested to fragments of the desired size (150-900 bp, equivalent to 1-5 nucleosomes). An antibody against endogenous c-Jun (Cell Signaling Technology, 9165) was used to immunoprecipitate complexes containing c-Jun. Following reversal of protein-DNA complexes and purification of DNA, RT-PCR was carried out as described above but using the purified DNA as a template. Three primer sets were designed to amplify $100-200 \mathrm{bp}$ fragments centred on the putative c-Jun binding site of the GLS promoter at position $-188 \mathrm{bp}$ relative to the TSS. Set 1 (forward and reverse): $5^{\prime}$-CCCTAGTACCCAACTAGGCTAGCC- $3^{\prime}$ and $5^{\prime}$-CCTCTCTTT TGATTGGCGATTAGGG-3'. Set 2 (forward and reverse): $5^{\prime}$-GCGTGCAGAAAG TGGCTACTGAGC- $3^{\prime}$ and ${ }^{\prime}$-CTCTCGGCTCTGGGTGCGCGGAGAG- $3^{\prime}$. Set 3 (forward and reverse): $5^{\prime}$-CCTCGGAGTTGGCACGGCGTGCAG- $3^{\prime}$ and $5^{\prime}$-GGCAGTCAAATTTCTCTCGGCTC-3'.

RNA interference. Knockdown of JUN expression in BT-549 cells was achieved using Silencer select pre-designed and validated siRNA (Life Technologies). Because endogenous c-Jun levels are so high in this cell line, two rounds of siRNA treatment were required to achieve effective knockdowns. Two siRNA products (manufacturer ID \# s7658 and s7659) were used, along with the Silencer select negative control no. 1 siRNA. Transfections were carried out in $10 \mathrm{~cm}$ dishes at $\sim 80 \%$ confluency using Lipofectamine 2000 transfection reagent (Life Technologies) and following the manufacturer's instructions. The final siRNA concentration was $15 \mathrm{nM}$ in all cases. Transfection medium was replaced with fresh growth medium $5 \mathrm{~h}$ after treatment. At $48 \mathrm{~h}$ following the initial transfection, the procedure was repeated, and cells were collected after a further $48 \mathrm{~h}$.

Stable cell lines. Transfection of breast cancer cell lines with plasmid DNA constructs (pCDNA3.1 or pCDNA3.1-JUN) was carried out using Lipofectamine 2000 transfection reagent (Life Technologies), following the manufacturer's instructions. At $48 \mathrm{~h}$ after transfection, cells were placed under G418 selection by supplementing the growth medium with G418 $\left(500 \mu \mathrm{g} \mathrm{ml}^{-1}\right.$ for MCF7, and $800 \mu \mathrm{g} \mathrm{ml}^{-1}$ for MDA-MB-468). Growth medium was replaced every $48 \mathrm{~h}$ for 2-3 weeks, until isolated colonies ( $\sim 2 \mathrm{~mm}$ diameter) were apparent on the plate. At this point, individual clones were transferred to 12 -well dishes and expanded in 
$250 \mu \mathrm{g} \mathrm{ml}^{-1} \mathrm{G} 418$ for further analysis. Appropriate clones were screened for c-Jun-V5 by western blot, and V5-positive clones were maintained in $250 \mu \mathrm{g} \mathrm{ml}^{-1}$ G418.

TCGA data. The Cancer Genome Atlas (TCGA) Breast Invasive Carcinoma (TCGA, provisional) data set was accessed, all data were analysed, and correlation plots prepared using the cBioportal ${ }^{45,46}$ suite of tools (www.cbioportal.org).

Statistical analyses. All differences were analysed with Student's $t$-test. A $P$ value $<0.05$ was considered to be significant and marked $\left.{ }^{*}\right)$, and a $P$ value below 0.01 was considered to be highly significant and marked $(* *)$.

\section{References}

1. DeBerardinis, R. J., Lum, J. J., Hatzivassiliou, G. \& Thompson, C. B. The biology of cancer: metabolic reprogramming fuels cell growth and proliferation. Cell Metab. 7, 11-20 (2008).

2. Dang, C. V. Links between metabolism and cancer. Genes Dev. 26, 877-890 (2012).

3. Lunt, S. Y. \& Vander Heiden, M. G. Aerobic glycolysis: meeting the metabolic requirements of cell proliferation. Annu. Rev. Cell Dev. Biol. 27, 441-464 (2011).

4. Tong, X., Zhao, F. \& Thompson, C. B. The molecular determinants of de novo nucleotide biosynthesis in cancer cells. Curr. Opin. Genet. Dev. 19, 32-37 (2009).

5. Lukey, M. J., Wilson, K. F. \& Cerione, R. A. Therapeutic strategies impacting cancer cell glutamine metabolism. Future Med. Chem. 5, 1685-1700 (2013).

6. Daye, D. \& Wellen, K. E. Metabolic reprogramming in cancer: Unraveling the role of glutamine in tumorigenesis. Semin. Cell Dev. Biol. 23, 362-369 (2012).

7. DeBerardinis, R. J. \& Cheng, T. Q's next: the diverse functions of glutamine in metabolism, cell biology and cancer. Oncogene 29, 313-324 (2010).

8. Cairns, R. A., Harris, I. S. \& Mak, T. W. Regulation of cancer cell metabolism. Nat. Rev. Cancer 11, 85-95 (2011).

9. Kroemer, G. \& Pouyssegur, J. Tumor cell metabolism: cancer's Achilles' heel. Cancer Cell 13, 472-482 (2008)

10. DeBerardinis, R. J. et al. Beyond aerobic glycolysis: transformed cells can engage in glutamine metabolism that exceeds the requirement for protein and nucleotide synthesis. Proc. Natl Acad. Sci. USA 104, 19345-19350 (2007).

11. Le, A. et al. Glucose-independent glutamine metabolism via TCA cycling for proliferation and survival in b cells. Cell Metab. 15, 110-121 (2012).

12. de la Rosa, V. et al. A novel glutaminase isoform in mammalian tissues. Neurochem. Int. 55, 76-84 (2009).

13. $\mathrm{Hu}, \mathrm{W}$. et al. Glutaminase 2, a novel p53 target gene regulating energy metabolism and antioxidant function. Proc. Natl Acad. Sci. USA 107, 7455-7460 (2010).

14. Cassago, A. et al. Mitochondrial localization and structure-based phosphate activation mechanism of Glutaminase $\mathrm{C}$ with implications for cancer metabolism. Proc. Natl Acad. Sci. USA 109, 1092-1097 (2012).

15. Mohamed, A., Deng, X., Khuri, F. R. \& Owonikoko, T. K. Altered glutamine metabolism and therapeutic opportunities for lung cancer. Clin. Lung Cancer 15, 7-15 (2014).

16. Huang, F., Zhang, Q., Ma, H., Lv, Q. \& Zhang, T. Expression of glutaminase is upregulated in colorectal cancer and of clinical significance. Int. J. Clin. Exp. Pathol. 7, 1093-1100 (2014).

17. Pan, T. et al. Elevated expression of glutaminase confers glucose utilization via glutaminolysis in prostate cancer. Biochem. Biophys. Res. Commun. 456, 452-458 (2015)

18. Szeliga, M. et al. Silencing of GLS and overexpression of GLS2 genes cooperate in decreasing the proliferation and viability of glioblastoma cells. Tumor Biol. 35, 1855-1862 (2014).

19. Robinson, M. M. et al. Novel mechanism of inhibition of rat kidney-type glutaminase by bis-2-(5-phenylacetamido-1,2,4-thiadiazol-2-yl)ethyl sulfide (BPTES). Biochem. J. 406, 407-414 (2007).

20. Wang, J. Bin et al. Targeting mitochondrial glutaminase activity inhibits oncogenic transformation. Cancer Cell 18, 207-219 (2010).

21. Gross, M. I. et al. Antitumor activity of the glutaminase inhibitor CB-839 in triple-negative breast cancer. Mol. Cancer Ther. 13, 890-901 (2014).

22. Wise, D. R. et al. Myc regulates a transcriptional program that stimulates mitochondrial glutaminolysis and leads to glutamine addiction. Proc. Natl Acad. Sci. USA 105, 18782-18787 (2008).

23. Gao, P. et al. c-Myc suppression of miR-23a/b enhances mitochondrial glutaminase expression and glutamine metabolism. Nature 458, 762-765 (2009).

24. Yuneva, M. O. et al. The metabolic profile of tumors depends on both the responsible genetic lesion and tissue type. Cell Metab. 15, 157-170 (2012).

25. Xia, Z. et al. Dynamic analyses of alternative polyadenylation from RNA-seq reveal a $3^{\prime}$-UTR landscape across seven tumour types. Nat. Commun. 5, 5274 (2014)
26. Bott, A. J. et al. Oncogenic Myc induces expression of glutamine synthetase through promoter demethylation. Cell Metab. 22, 1068-1077 (2015).

27. Qie, S., Chu, C., Li, W., Wang, C. \& Sang, N. ErbB2 activation upregulates glutaminase 1 expression which promotes breast cancer cell proliferation. J. Cell. Biochem. 115, 498-509 (2014).

28. Kenny, J. et al. Bacterial expression, purification, and characterization of rat kidney-type mitochondrial glutaminase. Protein Expr. Purif. 31, 140-148 (2003).

29. Angel, P., Hattori, K., Smeal, T. \& Karin, M. The jun proto-oncogene is positively autoregulated by its product, Jun/AP-1. Cell 55, 875-885 (1988).

30. Sabapathy, K. et al. Distinct roles for JNK1 and JNK2 in regulating JNK activity and c-Jun-dependent cell proliferation. Mol. Cell 15, 713-725 (2004).

31. Li, Z. et al. Regulation of PTEN by Rho small GTPases. Nat. Cell Biol. 7, 399-404 (2005)

32. Csibi, A. et al. The mTORC1/S6K1 pathway regulates glutamine metabolism through the eIF4B-dependent control of c-Myc translation. Curr. Biol. 24, 2274-2280 (2014).

33. Fanjul, A. et al. A new class of retinoids with selective inhibition of AP-1 inhibits proliferation. Nature 372, 107-111 (1994).

34. Lei, K. et al. The Bax subfamily of Bcl2-related proteins is essential for apoptotic signal transduction by c-Jun NH(2)-terminal kinase. Mol. Cell. Biol. 22, 4929-4942 (2002).

35. Zhao, C. et al. Genome-wide profiling of AP-1-regulated transcription provides insights into the invasiveness of triple-negative breast cancer. Cancer Res. 74, 3983-3994 (2014).

36. Vleugel, M. M., Greijer, A. E., Bos, R., van der Wall, E. \& van Diest, P. J. c-Jun activation is associated with proliferation and angiogenesis in invasive breast cancer. Hum. Pathol. 37, 668-674 (2006).

37. Shao, J. et al. COP1 and GSK3 $\beta$ cooperate to promote c-Jun degradation and inhibit breast cancer cell tumorigenesis. Neoplasia 15, 1075-1085 (2013).

38. Singh, R. et al. Differential effects of JNK1 and JNK2 inhibition on murine steatohepatitis and insulin resistance. Hepatology 49, 87-96 (2009).

39. Yang, G. et al. Isoform-specific palmitoylation of JNK regulates axonal development. Cell Death Differ. 19, 553-561 (2012).

40. Zhao, L. et al. Interferon- $\alpha$ regulates glutaminase 1 promoter through STAT1 phosphorylation: relevance to HIV-1 associated neurocognitive disorders. PLoS ONE 7, e32995 (2012).

41. Reynolds, M. R. et al. Control of glutamine metabolism by the tumor suppressor Rb. Oncogene 33, 556-566 (2014).

42. Messeguer, X. et al. PROMO: detection of known transcription regulatory elements using species-tailored searches. Bioinformatics 18, 333-334 (2002).

43. Koudritsky, M. \& Domany, E. Positional distribution of human transcription factor binding sites. Nucleic Acids Res. 36, 6795-6805 (2008).

44. Yang, W. et al. Genomics of drug sensitivity in cancer (GDSC): a resource for therapeutic biomarker discovery in cancer cells. Nucleic Acids Res. 41, D955-D961 (2013)

45. Cerami, E. et al. The cBio cancer genomics portal: an open platform for exploring multidimensional cancer genomics data. Cancer Discov. 2, 401-404 (2012).

46. Gao, J. et al. Integrative analysis of complex cancer genomics and clinical profiles using the cBioPortal. Sci. Signal. 6, pl1-pll (2013).

47. Florin, L. et al. Identification of novel AP-1 target genes in fibroblasts regulated during cutaneous wound healing. Oncogene 23, 7005-7017 (2004).

48. Eferl, R. \& Wagner, E. F. AP-1: a double-edged sword in tumorigenesis. Nat. Rev. Cancer 3, 859-868 (2003).

49. Shaulian, E. \& Karin, M. AP-1 as a regulator of cell life and death. Nat. Cell Biol. 4, E131-E136 (2002)

50. Cheng, T. et al. Pyruvate carboxylase is required for glutamine-independent growth of tumor cells. Proc. Natl Acad. Sci. USA 108, 8674-8679 (2011).

51. Stalnecker, C. A. et al. Mechanism by which a recently discovered allosteric inhibitor blocks glutamine metabolism in transformed cells. Proc. Natl Acad. Sci. USA 112, 394-399 (2014).

52. Maki, Y., Bos, T. J., Davis, C., Starbuck, M. \& Vogt, P. K. Avian sarcoma virus 17 carries the jun oncogene. Proc. Natl Acad. Sci. USA 84, 2848-2852 (1987).

53. Bohmann, D. et al. Human proto-oncogene c-jun encodes a DNA binding protein with structural and functional properties of transcription factor AP-1. Science 238, 1386-1392 (1987).

54. Schreiber, M. et al. Control of cell cycle progression by c-Jun is p53 dependent. Genes Dev. 13, 607-619 (1999).

55. Lallemand, D., Spyrou, G., Yaniv, M. \& Pfarr, C. M. Variations in Jun and Fos protein expression and AP-1 activity in cycling, resting and stimulated fibroblasts. Oncogene 14, 819-830 (1997).

56. Wada, T. et al. MKK7 couples stress signalling to G2/M cell-cycle progression and cellular senescence. Nat. Cell Biol. 6, 215-226 (2004).

57. Shaulian, E. \& Karin, M. AP-1 in cell proliferation and survival. Oncogene 20, 2390-2400 (2001).

58. Goodrich, D. W., Wang, N. P., Qian, Y. W., Lee, E. Y. \& Lee, W. H. The retinoblastoma gene product regulates progression through the G1 phase of the cell cycle. Cell 67, 293-302 (1991). 
59. Mariani, O. et al. JUN oncogene amplification and overexpression block adipocytic differentiation in highly aggressive sarcomas. Cancer Cell 11, 361-374 (2007).

60. Beroukhim, R. et al. The landscape of somatic copy-number alteration across human cancers. Nature 463, 899-905 (2010).

61. Kappelmann, M., Kuphal, S., Meister, G., Vardimon, L. \& Bosserhoff, A.-K. MicroRNA miR-125b controls melanoma progression by direct regulation of c-Jun protein expression. Oncogene 32, 2984-2991 (2013).

62. Bakiri, L., Matsuo, K., Wisniewska, M., Wagner, E. F. \& Yaniv, M. Promoter specificity and biological activity of tethered AP-1 dimers. Mol. Cell. Biol. 22, 4952-4964 (2002).

63. Mechta-Grigoriou, F., Gerald, D. \& Yaniv, M. The mammalian Jun proteins: redundancy and specificity. Oncogene 20, 2378-2389 (2001).

64. Smith, L. M. et al. cJun overexpression in MCF-7 breast cancer cells produces a tumorigenic, invasive and hormone resistant phenotype. Oncogene 18, 6063-6070 (1999)

65. Liu, Y. et al. AP-1 blockade in breast cancer cells causes cell cycle arrest by suppressing G1 cyclin expression and reducing cyclin-dependent kinase activity. Oncogene 23, 8238-8246 (2004).

66. Wang, X. et al. Elevated expression of phosphorylated c-Jun NH2-terminal kinase in basal-like and 'triple-negative' breast cancers. Hum. Pathol. 41, 401-406 (2010).

67. Horiuchi, D. et al. MYC pathway activation in triple-negative breast cancer is synthetic lethal with CDK inhibition. J. Exp. Med. 209, 679-696 (2012).

68. Wang, Z.-Y. et al. Regulation of IL-10 gene expression in Th2 cells by Jun proteins. J. Immunol. 174, 2098-2105 (2005).

\section{Acknowledgements}

We thank Cindy Westmiller for expert secretarial assistance. M.J.L. gratefully

acknowledges financial support from the Sam and Nancy Fleming Research Fellowship.
This work was supported by the National Institutes of Health grants (GM040654, GM047458 and CA163255).

\section{Author contributions}

M.J.L. conceived and designed the study, carried out experiments, analysed data and drafted the manuscript. K.S.G. prepared cDNAs, carried out RT-PCR experiments, carried out anchorage-independent growth assays and analysed data. J.W.E. and K.F.W provided conceptual advice, supplied reagents and contributed to the manuscript text. R.A.C. helped conceive and design the study, provided conceptual advice and contributed to the manuscript text. All authors read and approved the final manuscript.

\section{Additional information}

Supplementary Information accompanies this paper at http://www.nature.com/ naturecommunications

Competing financial interests: The authors declare no competing financial interests.

Reprints and permission information is available online at http://npg.nature.com/ reprintsandpermissions/

How to cite this article: Lukey, M. J. et al. The oncogenic transcription factor c-Jun regulates glutaminase expression and sensitizes cells to glutaminase-targeted therapy. Nat. Commun. 7:11321 doi: 10.1038/ncomms11321 (2016).

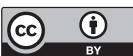

This work is licensed under a Creative Commons Attribution 4.0 International License. The images or other third party material in this article are included in the article's Creative Commons license, unless indicated otherwise in the credit line; if the material is not included under the Creative Commons license, users will need to obtain permission from the license holder to reproduce the material. To view a copy of this license, visit http://creativecommons.org/licenses/by/4.0/ 